\title{
Reduction of critical mass in a chemotaxis system by external application of a chemoattractant
}

\author{
José Ignacio Tello And Michael WinkLeR
}

\begin{abstract}
In this paper we study non-negative radially symmetric solutions of the parabolic-elliptic Keller-Segel system

$$
\begin{cases}u_{t}=\Delta u-\nabla \cdot(u \nabla v), & x \in \mathbb{R}^{2}, t>0, \\ 0=\Delta v+u+f_{0} \cdot \delta(x), & x \in \mathbb{R}^{2}, t>0,\end{cases}
$$

where $f_{0}>0$ and $\delta$ is the Dirac distribution. This system describes the chemotactic movement of cells under the additional circumstance that an external application of a chemoattractant at a distinguished point is introduced.

It is known that without such an external source the number $8 \pi$ plays the role of a critical mass in $(\star)$, in the sense that if the total mass $\mu:=\int_{\mathbb{R}^{2}} u_{0}$ of the cells exceeds $8 \pi$ then the solutions may blow up within finite time and collapse into a Dirac-type singularity, and that this does not occur when $\mu<8 \pi$.

The present paper shows that this critical number is reduced to $8 \pi-2 f_{0}$ by an application of the signal substance in the above way. Indeed, it is proved that whenever $f_{0}>0$ and $u_{0} \not \equiv 0$, a measure-valued global-in-time weak solution can be constructed which blows up at $x=0$ immediately. Now if $\mu<8 \pi-2 f_{0}$ then this solution satisfies $u(x, t) \leq C(\tau)|x|^{-\frac{f_{0}}{2 \pi}}$ for $t>\tau>0$ and $|x|<1$ and hence does not blow up in $L_{\mathrm{loc}}^{p}\left(\mathbb{R}^{2}\right)$ for any $1 \leq p<4 \pi / f_{0}$. On the other hand, if $\mu>8 \pi-f_{0}$ then the mass will asymptotically completely concentrate at the origin, that is, $u(\cdot, t)$ converges to $\mu \cdot \delta$ as $t \rightarrow \infty$ in the sense of Radon measures.
\end{abstract}

Mathematics Subject Classification (2010): 35K40 (primary); 35B44, 35K61 (secondary).

\section{Introduction}

Chemotaxis is the biological phenomenon whereby single-cells or multicellular organisms move guided by concentration gradients of a chemical. The response of the organisms to the chemical substance may be positive, leading to movement

The first author is partly supported by project MTM2009-13655 MICINN (Spain). Visiting ICMAT from june 2011 to May 2012.

Received June 21, 2011; accepted in revised form January 19, 2012. 
toward higher concentrations of the chemical, or negative, moving away from it. Chemotaxis appears in many different biological processes such as motion of bacteria, immune system response, migration of cells in embryonic development, and formation of blood vessels in tumor growth.

One of the first mathematical models which appears in the literature to describe aggregation of certain types of bacteria was proposed by Keller and Segel $[17,18]$, see also Patlak [26]. In the classical Keller-Segel system in the two-dimensional setting, the density distribution $u=u(x, t)$ of the living organisms is governed by the PDE

$$
u_{t}=\Delta u-\nabla \cdot(u \nabla v), \quad x \in \mathbb{R}^{2}, t>0,
$$

where $v$ represents the concentration of a chemoattractant substance. Secondly, in many biologically meaningful situations this chemoattractant is produced by the cells themselves (see [13] for a number of examples). Under the assumption that molecules diffuse much faster than cells, $v$ approximately satisfies an elliptic equation

$$
0=\Delta v+g(u, v), \quad x \in \mathbb{R}^{2}, t>0,
$$

where the function $g$ represents the balance of production and degradation of the signal substance.

In the pioneering work [16], the authors consider the particular case $g(u, v)=$ $u-\frac{1}{|\Omega|} \int_{\Omega} u_{0}$ in a circle $B_{0}(R) \subset \mathbb{R}^{2}$ of radius $R$ and they prove that radially symmetric solutions exist globally and remain bounded if the total mass $\int_{\Omega} u_{0}$ of cells is small, whereas some solutions may blow up in finite time with respect to the norm in $L^{\infty}(\Omega)$ when $\int_{\Omega} u_{0}$ is suitably large. This critical mass phenomenon, already conjectured in [8], attracted a considerable interest in the subsequent years, and quite a complete answer is available in the radial version of the corresponding Cauchy problem in the whole space $\mathbb{R}^{2}$ where accordingly $g(u, v)=u$. In fact, in this framework the number $8 \pi$ plays the role of a critical mass in the sense that whenever $\int_{\mathbb{R}^{2}} u_{0}<8 \pi$, the solution is global and bounded, whereas if $\int_{\mathbb{R}^{2}} u_{0}>8 \pi$ then the solution will blow up in finite time. A comprehensive demonstration of this, using approaches originating from [22], can be found in [1]. In addition, a rich literature has revealed that a critical mass phenomenon also occurs in a number of related chemotaxis systems, both of parabolic-elliptic and of parabolic-parabolic type, and also in non-radial frameworks and in corresponding Neumann-type boundaryvalue problems. Here the main difference arising in the non-symmetric bounded domain case appears to be the fact that the mass threshold is then halved to $4 \pi$, which is due to the possible occurrence of blow-up on the boundary of the domain (see $[2,6,20,23]$ for parabolic-elliptic systems and $[9,15,24,28]$ for fully parabolic analogues).

As to the qualitative behavior of such large mass solutions near blow-up, formal arguments suggest that the cell population aggregates at the origin in the strongest conceivable sense, namely, that the spatial profile of the first component 
$u$ of an unbounded solution should approach a Dirac mass near its blow-up time (see [34]). In the borderline case $\int_{\mathbb{R}^{2}} u_{0}=8 \pi$, such a collapse into a Dirac measure occurs in infinite time, as was proved in [7]. Some further results on blow-up mechanisms in this and related Keller-Segel systems support the conjecture that this tendency toward Dirac-type singularity formation in fact is a generic phenomenon (see $[11,12]$ and $[31,32]$ as well as [33] for a simplified argument).

It is the purpose of the present work to examine in how far these mechanisms are influenced by an external source of chemoattractant. The motivational background for this stems from the observation that artificial gradients of chemoattractants are frequently introduced in experiments, see for instance [37] where corresponding effects on the migration of hematopoietic progenitor cells are studied.

As a prototypical model for such a process, subsequently we shall consider the problem

$$
\begin{cases}u_{t}=\Delta u-\nabla \cdot(u \nabla v), & x \in \mathbb{R}^{2}, t>0, \\ 0=\Delta v+u+f_{0} \delta(x), & x \in \mathbb{R}^{2}, t>0, \\ u(x, 0)=u_{0}(x), & x \in \mathbb{R}^{2},\end{cases}
$$

with a constant $f_{0}>0$, where $u_{0} \neq \equiv 0$ is a given radially symmetric bounded non-negative function with finite total mass, that is,

$$
\mu:=\int_{\mathbb{R}^{2}} u_{0}(x) d x<\infty .
$$

Here, $\delta$ denotes the Dirac distribution supported at the origin, and thus $(0.1)$ describes the respective evolution when the chemoattractant is introduced precisely at the spatial origin, with constant rate $f_{0}$. In this framework, the question we address is the following:

\section{Does the source term $f_{0} \cdot \delta(x)$ reduce the threshold number $8 \pi$ for chemotactic aggregation into a Dirac singularity?}

Our main results in this direction state that the critical mass is actually reduced to

$$
\mu_{c}:=8 \pi-2 f_{0},
$$

but as compared to the borderline case $f_{0}=0$ this threshold has a slightly different meaning. Indeed, we shall first prove that for any choice of $f_{0}>0$, a global solution of (0.1) exists in some generalized sense ( $c f$. Definition 1.1); however, any such solution blows up immediately:

Theorem 0.1. Let $f_{0} \geq 0$. Then for all $t_{0} \geq 0$ there exists a globally defined radial weak solution $u$ of $(0.1)$. If $f_{0}>0$, then this solution satisfies

$$
\|u\|_{L^{\infty}\left(\mathbb{R}^{2} \times\left(t_{0}, t_{0}+\tau\right)\right)}=\infty \quad \text { for all } \tau>0 .
$$

Now the size of the initial mass, as compared to $\mu_{c}$, decides whether the above solution approaches a Dirac singularity, or remains less singular: 


\section{Theorem 0.2.}

i) Assume that $f_{0} \geq 0$, and that $u_{0}$ satisfies (0.2) with some $\mu>8 \pi-2 f_{0}$. Then the radial weak solution $u$ from Theorem 0.1 satisfies

$$
u(x, t) \stackrel{\star}{\rightarrow} \mu \delta(x) \quad \text { as } t \rightarrow \infty
$$

in the sense of Radon measures over $\mathbb{R}^{2}$.

ii) Suppose that $f_{0} \in[0,4 \pi)$, and that $u_{0}$ satisfies $(0.2)$ with some $\mu<8 \pi-2 f_{0}$. Then for all $\tau>0$ there exists $C(\tau)>0$ such that for the radial weak solution $u$ from Theorem 0.1 we have

$$
u(x, t) \leq C(\tau)|x|^{-\frac{f_{0}}{2 \pi}} \quad \text { for all } x \in B_{1}(0) \text { and } t \geq \tau .
$$

In particular, given any $p \in\left[1, \frac{4 \pi}{f_{0}}\right)$ and $\tau>0$ one can find $C(\tau)>0$ with the property that

$$
\|u(\cdot, t)\|_{L^{p}\left(B_{1}(0)\right)} \leq C(\tau) \quad \text { for all } t \geq \tau .
$$

Theorems 0.1 and 0.2 can be also applied to the case $f_{0}=0$ to obtain results similar to those derived in [2], where finite-time blow-up and continuation of solutions for the supercritical case were described. In this context we also refer to [30], where certain global weak solutions for the case $f_{0}=0$ were constructed. We also mention the recent work [5] which is related to our Theorem 0.1 in that it can be used to state a nonexistence result for classical, and also for some weak, solutions of (1.2) in any time interval when $f_{0}>0$. However, this apparently does not immediately entail that $(0.3)$ is valid for the (very weak) solution that we will obtain.

This study is to be understood as a first step towards understanding the behavior of chemotactic movement under the influence of external sources. One mathematically interesting open problem is to clarify the behavior in the critical-mass case $\mu=8 \pi-f_{0}$ when $f_{0}>0$. Also, allowing for more general source terms $f(x)$, not necessarily in the radial setting, would be worthwhile being studied, because it might be thought of as a preparatory step for the corresponding optimal control problem which targets at approaching a desired distribution of cells after a given time by suitably adjusting an external application of signal.

Let us remark that clearly $v$ can be expected to be unique only up to addition of constants. However, since we do not address the uniqueness question for (0.1) in this paper, we refrain from introducing an extra normalization condition for $v$ such as $\int_{B_{1}(0)} v \equiv 0$, because this evidently does not change any of the claimed results. unknown

The paper is organized as follows. In Section 1 the auxiliary problem for the

$$
W(s, t):=2 \int_{0}^{\sqrt{s}} r u(r, t) d r, \quad s>0, t>0
$$

is introduced and preliminary properties of $W$ are studied. In Section 2 we present the proof of instantaneous blow-up for $f_{0}>0$ (Theorem 0.1). In Section 3 we address the problem for supercritical mass (Theorem 0.2 (i)). The last section is devoted to the analysis of the subcritical case (Theorem 0.2 (ii)). 


\section{Existence of measure-valued solutions}

Following [16], we transform (0.1) by introducing

$$
W(s, t):=\frac{1}{\pi} \int_{B_{\sqrt{s}}(0)} u(x, t) d x, \quad s>0, t>0,
$$

which in the case of a radially symmetric $u=u(r, t)$ means that we set

$$
W(s, t)=2 \int_{0}^{\sqrt{s}} r u(r, t) d r, \quad s>0, t>0 .
$$

We are thereby formally led to considering the degenerate parabolicinitial-boundary value problem

$$
\begin{cases}W_{t}=4 s W_{s s}+W W_{s}+2 F_{0} W_{s}, & s>0, t>0, \\ W(0, t)=0, & \lim _{s \rightarrow \infty} W(s, t)=\frac{\mu}{\pi}, \quad t>0 \\ W(s, 0)=W_{0}(s), & s>0,\end{cases}
$$

with parameter

$$
F_{0}:=\frac{f_{0}}{2 \pi}
$$

and initial data

$$
W_{0}(s):=2 \int_{0}^{\sqrt{s}} \rho u_{0}(\rho) d \rho, \quad s>0 .
$$

Observe that if $u_{0}$ is non-negative and bounded fulfilling (0.2) then $W_{0}$ satisfies

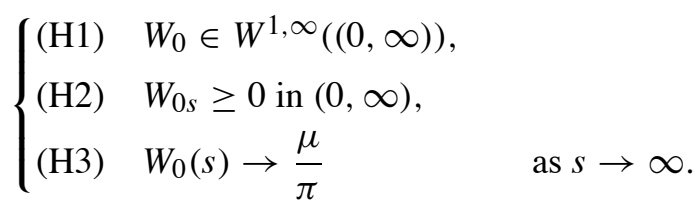

Clearly, in view of (1.1) for each $t$ the function $W(\cdot, t)$ must be non-decreasing, and if $u$ enjoys the expected mass-conservation property $\int_{\mathbb{R}^{2}} u(x, t) d x \equiv \mu$ then $W(s, t) \rightarrow \frac{\mu}{\pi}$ as $s \rightarrow \infty$. In particular, this means that $W$ is bounded and hence standard parabolic regularity theory tells us that $W_{s}$ is smooth in $(0, \infty) \times(0, \infty)$, because the PDE in (1.2) is uniformly parabolic in each cylinder $\left(s_{0}, \infty\right) \times(0, \infty)$ with $s_{0}>0$. As a consequence, away from the origin we expect the pointwise identity $W_{s}(s, t)=u(\sqrt{s}, t)$ suggested by (1.1), and the only possibility for $u(\cdot, t)$ to develop a singularity corresponds to a jump of $W(\cdot, t)$ at $s=0$ with jump size determined by $W(0+, t):=\lim _{s \searrow 0} W(s, t)$. Thus, once we are given a possibly 
discontinuous solution $W$ of (1.2) we can reconstruct $u$ according to the measurevalued identity

$$
u(x, t):=W_{s}\left(|x|^{2}, t\right)+\pi W(0+, t) \cdot \delta(x)
$$

for $t>0$. For the study of global-in-time solutions of $(0.1)$ it therefore appears to be natural to act in the framework of Radon measures. To become more precise in the following definition, we let $\mathcal{M}_{\mathrm{rad}}\left(\mathbb{R}^{2}\right)$ denote the space of radially symmetric Radon measures on $\mathbb{R}^{2}$, that is, the space of all functionals $\lambda$, radially symmetric about $x=0$, defined on the space $C_{0}^{0}\left(\mathbb{R}^{2}\right)$ of compactly supported continuous functions over $\mathbb{R}^{2}$. Here we recall that such a functional $\lambda$ is radially symmetric about $x=0$ if $\lambda\left(\frac{\partial \psi}{\partial \phi}\right)=0$ for all $\psi=\psi(r, \phi) \in C_{0}^{\infty}\left(\mathbb{R}^{2}\right)$.

Definition 1.1. Let $f_{0} \geq 0$, and assume that $u_{0} \in L^{\infty}\left(\mathbb{R}^{2}\right)$ is non-negative and $\mu:=\int_{\mathbb{R}^{2}} u_{0}$ is finite. Then by a radial weak solution of $(0.1)$ in $\mathbb{R}^{2} \times(0, \infty)$ we mean a function

$$
u \in C^{0}\left([0, \infty) ; \mathcal{M}_{\mathrm{rad}}\left(\mathbb{R}^{2}\right)\right)
$$

such that the function $W:[0, \infty) \times[0, \infty)$ defined by (1.1) satisfies $W(s, t) \rightarrow \frac{\mu}{\pi}$ as $s \rightarrow \infty$ for all $t>0$ and

$$
\begin{aligned}
& -\int_{0}^{\infty} \int_{0}^{\infty} \zeta_{t} W-\int_{0}^{\infty} \zeta(\cdot, 0) W_{0} \\
& =4 \int_{0}^{\infty} \int_{0}^{\infty}(s \zeta)_{s s} W-\frac{1}{2} \int_{0}^{\infty} \int_{0}^{\infty} \zeta_{s} W^{2}-\frac{f_{0}}{2 \pi} \int_{0}^{\infty} \int_{0}^{\infty} \zeta_{s} W
\end{aligned}
$$

for all $\zeta \in C_{0}^{\infty}([0, \infty) \times[0, \infty))$, where $W_{0}(s):=\frac{1}{\pi} \int_{B_{\sqrt{s}}(0)} u_{0}(x) d x$ for $s \geq 0$.

In order to construct such solutions of (1.2) by a suitable approximation procedure, we fix a cut-off function $\chi \in C^{\infty}([0, \infty))$ such that $\chi \equiv 0$ on $\left[0, \frac{1}{2}\right], \chi \equiv 1$ on $[1, \infty)$ and $\chi^{\prime} \geq 0$ on $[0, \infty)$, and for $\varepsilon \in(0,1)$ we introduce $\chi^{(\varepsilon)}(s):=\chi\left(\frac{s}{\varepsilon}\right)$, $s \geq 0$. Then

$$
\chi^{(\varepsilon)} \equiv 0 \text { on }\left[0, \frac{\varepsilon}{2}\right], \quad \chi^{(\varepsilon)} \equiv 1 \text { on }[\varepsilon, \infty) \quad \text { and } \quad \chi_{s}^{(\varepsilon)} \geq 0 \text { on }[0, \infty),
$$

and

$$
\left|\chi_{s}^{(\varepsilon)}\right| \leq \frac{c_{\chi}}{\varepsilon} \quad \text { and } \quad\left|\chi_{s s}^{(\varepsilon)}\right| \leq \frac{c_{\chi}}{\varepsilon^{2}}
$$

are valid with $c_{\chi}:=\left\|\chi^{\prime}\right\|_{L^{\infty}((0, \infty))}+\left\|\chi^{\prime \prime}\right\|_{L^{\infty}((0, \infty))}$. Moreover,

$$
\chi^{(\varepsilon)}(s) \nearrow 1 \quad \text { as } \varepsilon \rightarrow 0
$$

holds for all $s>0$. 
We first make sure that the approximate problems

$$
\begin{cases}W_{t}^{(\varepsilon)}=4 s W_{s s}^{(\varepsilon)}+\chi^{(\varepsilon)}(s) W^{(\varepsilon)} W_{s}^{(\varepsilon)}+2 F_{0} \chi^{(\varepsilon)}(s) W_{s}^{(\varepsilon)}, & s>0, t>0, \\ W^{(\varepsilon)}(0, t)=0, \quad \lim _{s \rightarrow \infty} W^{(\varepsilon)}(s, t)=\frac{\mu}{\pi}, & t>0, \\ W^{(\varepsilon)}(s, 0)=W_{0}(s), & s>0,\end{cases}
$$

allow for a comparison principle:

Lemma 1.2. Let $F_{0} \geq 0$ and $\varepsilon>0$, and assume that $\bar{W}$ and $\underline{W}$ belong to $C^{0}([0, \infty) \times$ $[0, \infty) \cap C^{2,1}((0, \infty) \times(0, \infty))$ and satisfy

$$
\bar{W}_{t} \geq 4 s \bar{W}_{s s}+\chi^{(\varepsilon)}(s) \overline{W W}_{s}+2 F_{0} \chi^{(\varepsilon)}(s) \bar{W}_{s}
$$

and

$$
\underline{W}_{t} \leq 4 s \underline{W}_{s s}+\chi^{(\varepsilon)}(s) \underline{W}_{s}+2 F_{0} \chi^{(\varepsilon)}(s) \underline{W}_{s}
$$

for all $s>0$ and $t>0$. Moreover, suppose that the initial data $\bar{W}_{0}:=\bar{W}(\cdot, 0)$ and $\underline{W}_{0}:=\underline{W}(\cdot, 0)$ satisfy $(1.3)$ with positive numbers $\bar{\mu}$ and $\underline{\mu}$, respectively, and that $\bar{W}_{0} \geq \underline{W}_{0}$ holds on $(0, \infty)$. Then $\bar{W} \geq \underline{W}$ in $[0, \infty) \times[0, \infty)$.

Proof. According to our hypotheses, $d:=\underline{W}-\bar{W}$ satisfies

$$
d(0, t) \leq 0 \quad \text { and } \quad \lim _{s \rightarrow \infty} d(s, t) \leq 0 \quad \text { for all } t>0
$$

and

$$
d(s, 0) \leq 0 \quad \text { for all } s>0,
$$

and subtracting (1.9) from (1.10) yields

$$
d_{t} \leq 4 s d_{s s}+\frac{1}{2} \chi^{(\varepsilon)} \cdot\left(\underline{W}^{2}-\bar{W}^{2}\right)_{s}+2 F_{0} \chi^{(\varepsilon)} d_{s} \quad \text { for all } s>0 \text { and } t>0 .
$$

Let $\xi(d):=(d-\gamma)_{+}$for $\gamma>0$; then, multiplying by $\frac{\xi(d)}{s}$ and integrating by parts gives

$$
\begin{aligned}
& \frac{1}{2} \frac{d}{d t} \int_{0}^{\infty} \frac{\xi^{2}(d)}{s}=-4 \int_{0}^{\infty}(\xi(d))_{s}^{2}-\frac{1}{2} \int_{0}^{\infty} \frac{\chi^{(\varepsilon)}(s)}{s}\left(\underline{W}^{2}-\bar{W}^{2}\right) \cdot(\xi(d))_{s} \\
& +\frac{1}{2} \int_{0}^{\infty} \frac{\chi^{(\varepsilon)}(s)}{s^{2}} \cdot\left(\underline{W}^{2}-\bar{W}^{2}\right) \cdot \xi(d)-\frac{1}{2} \int_{0}^{\infty} \frac{\chi_{s}^{(\varepsilon)}(s)}{s} \cdot\left(\underline{W}^{2}-\bar{W}^{2}\right) \cdot \xi(d) \\
& +F_{0} \int_{0}^{\infty} \frac{\chi^{(\varepsilon)}(s)}{s^{2}} \cdot \xi^{2}(d)-F_{0} \int_{0}^{\infty} \frac{\chi_{s}^{(\varepsilon)}(s)}{s} \cdot \xi^{2}(d) \quad \text { for all } t>0
\end{aligned}
$$


Here the fourth and the last term on the right are non-negative, because $\chi_{s}^{(\varepsilon)} \geq 0$ and $\underline{W}^{2}>\bar{W}^{2}$ whenever $\xi(d)>0$. Next, by Young's inequality we have

$$
\begin{aligned}
& -\frac{1}{2} \int_{0}^{\infty} \frac{\chi^{(\varepsilon)}(s)}{s} \cdot\left(\underline{W}^{2}-\bar{W}^{2}\right)\left(\xi^{2}(d)\right)_{s} \\
& \leq 4 \int_{0}^{\infty}(\xi(d))_{s}^{2}+\frac{1}{64} \int_{\frac{\varepsilon}{2}}^{\infty} \frac{1}{s^{2}} \cdot\left(\underline{W^{2}}-\bar{W}^{2}\right)^{2} \cdot \chi\{d>\gamma\}
\end{aligned}
$$

and

$$
\frac{1}{2} \int_{0}^{\infty} \frac{\chi^{(\varepsilon)}(s)}{s^{2}}\left(\underline{W^{2}}-\bar{W}^{2}\right) \xi(d) \leq \frac{1}{4} \int_{0}^{\infty} \frac{\xi^{2}(d)}{s}+\frac{1}{4} \int_{\frac{\varepsilon}{2}}^{\infty} \frac{1}{s^{3}} \cdot\left(\underline{W^{2}}-\bar{W}^{2}\right)^{2} \cdot \chi_{\{d>\gamma\}},
$$

for $\chi^{(\varepsilon)} \equiv 0$ on $\left[0, \frac{\varepsilon}{2}\right]$ and $0 \leq \chi^{(\varepsilon)} \leq 1$. By means of the estimate

$$
\left(\underline{W}^{2}-\bar{W}^{2}\right)^{2}=(\underline{W}+\bar{W})^{2} d^{2} \leq\left(\frac{\underline{\mu}+\bar{\mu}}{\pi}\right)^{2} \cdot d^{2} \leq c_{1}\left((d-\gamma)_{+}^{2}+\gamma^{2}\right) \quad \text { in }\{d>\gamma\},
$$

valid for $c_{1}:=2\left(\frac{\mu+\bar{\mu}}{\pi}\right)^{2}$, from (1.12) we thereby obtain

$$
\begin{aligned}
\frac{1}{2} \frac{d}{d t} \int_{0}^{\infty} \frac{\xi^{2}(d)}{s} \leq & \frac{c_{1}}{64} \int_{\frac{\varepsilon}{2}}^{\infty} \frac{\xi^{2}(d)}{s^{2}}+\frac{c_{1}}{4} \int_{\frac{\varepsilon}{2}}^{\infty} \frac{\xi^{2}(d)}{s^{3}}+\frac{1}{4} \int_{0}^{\infty} \frac{\xi^{2}(d)}{s} \\
& +F_{0} \int_{\frac{\varepsilon}{2}}^{\infty} \frac{\xi^{2}(d)}{s^{2}}+\frac{c_{1} \gamma^{2}}{64} \int_{\frac{\varepsilon}{2}}^{\infty} \frac{d s}{s^{2}}+\frac{c_{1} \gamma^{2}}{4} \int_{\frac{\varepsilon}{2}}^{\infty} \frac{d s}{s^{3}} \\
\leq & c_{2} \int_{0}^{\infty} \frac{\xi^{2}(d)}{s}+c_{2} \gamma^{2} \quad \text { for all } t>0,
\end{aligned}
$$

where $c_{2}$ is a positive constant possibly depending on $\varepsilon$ but not on $\gamma$. It remains to integrate the resulting ODE, to recall (1.11) and to let $\gamma \rightarrow 0$ to infer that $d_{+} \equiv 0$, which implies the claim.

We next prove that the approximate problem (1.8) is uniquely solvable in the classical sense:

Lemma 1.3. Assume that $F_{0} \geq 0$, and that $W_{0}$ satisfies (1.3) with some $\mu>0$. Then for each $\varepsilon \in(0,1)$ there exists precisely one function

$$
W^{(\varepsilon)} \in C^{0}([0, \infty) \times[0, \infty)) \cap C^{2,1}((0, \infty) \times(0, \infty))
$$

which satisfies (1.8) in the classical sense. 
Proof. Since uniqueness trivially results from Lemma 1.2, we only need to prove existence of a classical solution. To achieve this, for $S>0$ we consider the degenerate parabolic problem on a bounded interval,

$$
\begin{cases}W_{t}^{(\varepsilon, S)}=4 s W_{s s}^{(\varepsilon, S)}+\chi^{(\varepsilon)}(s) W^{(\varepsilon, S)} W_{s}^{(\varepsilon, S)} & \\ \quad+2 F_{0} \chi^{(\varepsilon)}(s) W_{s}^{(\varepsilon, S)}, & s \in(0, S), t>0, \\ W^{(\varepsilon, S)}(0, t)=0, \quad W^{(\varepsilon, S)}(S, t)=W_{0}(S), & t>0, \\ W^{(\varepsilon, S)}(s, 0)=W_{0}(s), & s \in(0, S),\end{cases}
$$

and the uniformly parabolic regularizations thereof given by

$$
\left\{\begin{array}{rlrl}
W_{t}^{(\varepsilon, S, \eta)}= & 4(s+\eta) W_{s s}^{(\varepsilon, S, \eta)} & & \\
& +\chi^{(\varepsilon)}(s) W^{(\varepsilon, S, \eta)} W_{s}^{(\varepsilon, S, \eta)} & & \\
& +2 F_{0} \chi^{(\varepsilon)}(s) W_{s}^{(\varepsilon, S, \eta)}, & & s \in(0, S), t>0 \\
& W^{(\varepsilon, S, \eta)}(0, t)=0, \quad W^{(\varepsilon, S, \eta)}(S, t)=W_{0}(S), & & t>0, \\
W^{(\varepsilon, S, \eta)}(s, 0)=W_{0}(s), & & s \in(0, S)
\end{array}\right.
$$

where $\eta \in(0,1)$. By standard parabolic theory, (1.14) admits a classical solution $W^{(\varepsilon, S, \eta)} \in C^{0}([0, S] \times[0, \infty)) \cap C^{2,1}([0, S] \times(0, \infty))$, which is global in time and bounded, because the inequalities $0 \leq W_{0} \leq \frac{\mu}{\pi}$ along with the maximum principle entail the uniform two-sided a priori bound $0 \leq W^{(\varepsilon, S, \eta)} \leq \frac{\mu}{\pi}$. In conjunction with parabolic regularity theory ([21]), this moreover provides uniform estimates for the family $\left(W^{(\varepsilon, S, \eta)}\right)_{\eta \in(0,1)}$ in the spaces $C_{\mathrm{loc}}^{\theta, \frac{\theta}{2}}((0, S] \times[0, \infty))$ and $C_{\mathrm{loc}}^{2+\theta, 1+\frac{\theta}{2}}((0, S] \times$ $(0, \infty))$ for some $\theta>0$. Thus, the Arzelà-Ascoli theorem enables us to extract a sequence of numbers $\eta_{j} \searrow 0$ such that $W^{(\varepsilon, S, \eta)} \rightarrow W^{(\varepsilon, S)}$ in $C_{\text {loc }}^{0}((0, S] \times$ $[0, \infty) \cap \cap C_{\mathrm{loc}}^{2,1}((0, S] \times(0, \infty))$ as $\eta=\eta_{j} \rightarrow 0$ for some limit function $W^{(\varepsilon, S)}$ which can easily be seen to satisfy the first and third lines in (1.13) as well as $W^{(\varepsilon, S)}(S, t)=W_{0}(S, t)$ for all $t>0$. In order to obtain continuity also down to $s=0$, we use that by (1.6) and (1.7), $\chi^{(\varepsilon)}(s) \leq \frac{c_{\chi} s}{\varepsilon}$ for all $s \geq 0$ and $\varepsilon \in(0,1)$, whence

$$
W_{t}^{(\varepsilon, S, \eta)} \leq 4(s+\eta) W_{s s}^{(\varepsilon, S, \eta)}+C_{\varepsilon} s W_{s}^{(\varepsilon, S, \eta)}
$$

holds in $(0, S) \times(0, \infty)$ with $C_{\varepsilon}:=\frac{c_{\chi}}{\varepsilon}\left(\frac{\mu}{\pi}+2 F_{0}\right)$, because $W^{(\varepsilon, S, \eta)} \leq \frac{\mu}{\pi}$. Using $\bar{W}(s, t):=c_{1} e^{C_{\varepsilon} t} \cdot s$ with $c_{1}:=\left\|W_{0 s}\right\|_{L^{\infty}((0, \infty))}$ as an upper comparison function here, we find that

$$
\begin{aligned}
& W^{(\varepsilon, S, \eta)}(s, t) \leq c_{1} e^{C_{\varepsilon} t} \cdot s \quad \text { in }[0, \infty) \times[0, \infty) \text { for all } \varepsilon \in(0,1), S>0 \\
& \text { and } \eta \in(0,1) .
\end{aligned}
$$

Accordingly, the convergence $W^{(\varepsilon, S, \eta)} \rightarrow W^{(\varepsilon, S)}$ is actually locally uniform in $[0, \infty) \times[0, \infty)$ and $W^{(\varepsilon, S)}$ also satisfies the desired boundary condition $W^{(\varepsilon, S)}(0, t)=$ 0 for all $t>0$. 
Next, since (1.15) implies that $W^{(\varepsilon, S)}(s, t) \leq c_{1} e^{C_{\varepsilon} t} \cdot s$ in $[0, \infty) \times[0, \infty)$ for all $\varepsilon \in(0,1)$ and $S>0$, we may follow a similar reasoning to find a sequence of numbers $S_{k} \rightarrow \infty$ such that $W^{\left(\varepsilon, S_{k}\right)} \rightarrow W^{(\varepsilon)}$ in $C_{\mathrm{loc}}^{0}([0, \infty) \times[0, \infty)) \cap$ $C_{\text {loc }}^{2,1}((0, \infty) \times(0, \infty))$ as $S_{k} \rightarrow \infty$, where $W^{(\varepsilon)}$ is a non-negative function solving (1.8) in the classical sense. Note here that since $W_{0 s} \geq 0$, the maximum principle ensures that $W_{S}^{(\varepsilon, S, \eta)} \geq 0$ in $(0, S) \times(0, \infty)$ for all $\varepsilon \in(0,1), S>0$ and $\eta \in(0,1)$ and hence $\lim _{s \rightarrow \infty} W^{(\varepsilon)}(s, t)$ exists and coincides with $\lim _{S=S_{k} \rightarrow \infty} W^{(\varepsilon, S)}(S, t)=$ $\lim _{k \rightarrow \infty} W_{0}\left(S_{k}\right)=\frac{\mu}{\pi}$ for all $t>0$.

We can proceed to prove global existence of a weak solution of the original problem in the radial framework:

Lemma 1.4. Let $f_{0} \geq 0$ and assume that $u_{0} \in L^{\infty}\left(\mathbb{R}^{2}\right)$ is radially symmetric, non-negative and satisfies (0.2). Then there exists at least one radial weak solution $u$ of $(0.1)$ in the sense of Definition 1.1. More precisely, the solutions $W^{(\varepsilon)}$ of (1.8) increase to a limit $W$ as $\varepsilon \searrow 0$, and $u$ as defined by (1.4) solves (0.1) in the claimed sense.

Proof. Using that $\chi^{(\varepsilon)} \nearrow 1$ in $(0, \infty)$ as $\varepsilon \searrow 0$, we see by comparison that $W^{(\varepsilon, S, \eta)}$ is nonincreasing with respect to $\varepsilon \in(0,1)$. Accordingly, we have

$$
W^{(\varepsilon)} \nearrow W \quad \text { in }(0, \infty) \times(0, \infty) \quad \text { as } \varepsilon \searrow 0
$$

with some limit function $W$ which, as an easy consequence, satisfies the boundary condition $W(s, t) \rightarrow \frac{\mu}{\pi}$ as $s \rightarrow \infty$ for all $t>0$. Moreover, if we fix $\zeta \in C_{0}^{\infty}([0, \infty) \times[0, \infty))$ and test $(1.8)$ by $\zeta$ to obtain an obvious analogue of (1.5) for $\varepsilon>0$, by (1.16) we can take $\varepsilon \searrow 0$ in each integral to arrive at (1.5) in the limit.

Adopting a notion introduced in [10], in the sequel we shall call the function $W$ defined through (1.16) the proper solution of (1.2).

Applying Lemma 1.2 to appropriate solutions of (1.8) and taking limits, we easily obtain the following favorable comparison property of proper solutions:

Lemma 1.5. Suppose that $F_{0} \geq 0$ and that $\bar{W}$ and $\underline{W}$ are proper solutions emanating from $\bar{W}_{0}$ and $\underline{W}_{0}$, where $\bar{W}_{0}$ and $\underline{W}_{0}$ satisfy (1.3) with certain $\bar{\mu}>0$ and $\underline{\mu}>0$, respectively. If $\bar{W}_{0} \geq \underline{W}_{0}$ in $[0, \infty)$ then $\bar{W} \geq \underline{W}$ in $[0, \infty) \times[0, \infty)$.

For later use, let us provide a statement on time monotonicity of some proper solutions of (1.2):

Lemma 1.6. Assume that $F_{0} \geq 0$ and that $W_{0}$ satisfies (1.3). Moreover, suppose that there exists $s_{0}>0$ such that $W_{0} \equiv 0$ in $\left[0, s_{0}\right]$, that $W_{0} \in C^{2}\left(\left[s_{0}, \infty\right)\right)$ with $\liminf _{s \searrow s_{0}} W_{0 s}(s)>0$, and that

$$
4 s W_{0 s s}+W_{0} W_{0 s}+2 F_{0} W_{0 s} \geq 0 \quad \text { in }\left(s_{0}, \infty\right) .
$$

Then the proper solution $W$ of (1.2) fulfils

$$
W_{t} \geq 0 \quad \text { in }(0, \infty) \times(0, \infty) .
$$


Proof. By construction of $\chi^{(\varepsilon)}$, for all sufficiently small $\varepsilon>0$ we have

$$
I(s):=4 s W_{0 s s}+\chi^{(\varepsilon)} W_{0} W_{0 s}+2 F_{0} \chi^{(\varepsilon)} W_{0 s} \geq 0
$$

in the sense of distributions over $(0, \infty)$, because $I(s)=0$ for all $s \in\left(0, s_{0}\right) \supset$ $(0, \varepsilon)$, since $I(s) \geq 0$ for all $s \in\left(s_{0}, \infty\right)$ by $(1.17)$, and because $\liminf _{s \backslash s_{0}} W_{0 s}(s)>$ $0=\lim \sup _{s \nearrow s_{0}} W_{0 s}(s)$. Therefore, a standard reasoning (see [27, Ch. 52]) allows us to conclude that the solutions $W^{(\varepsilon)}$ of $(1.8)$ satisfy $W_{t}^{(\varepsilon)} \geq 0$ in $(0, \infty) \times(0, \infty)$ for all $\varepsilon \in(0,1)$, from which (1.18) easily follows.

\section{Instantaneous blow-up}

It is the purpose of the present section to make sure that, in a sense to be specified below, the spatial derivative $W_{s}$ of the proper solution $W$ of (1.2) blows up immediately, and that hence so does $u$. In order to prepare this, we state two auxiliary lemmata, the first of which can be regarded as a variant of Gronwall's lemma:

Lemma 2.1. Suppose that $\Phi \in W_{\text {loc }}^{1, \infty}(\mathbb{R})$ is non-decreasing, and that for some $t_{1} \in \mathbb{R}, T>0$ and $c \in \mathbb{R}$ we are given two functions $y \in C^{0}\left(\left[t_{1}, t_{1}+T\right)\right)$ and $z \in C^{1}\left(\left[t_{1}, t_{1}+T\right)\right)$ such that

$$
y(t) \geq c+\int_{t_{1}}^{t} \Phi(y(\bar{t})) d \bar{t} \quad \text { for all } t \in\left(t_{1}, t_{1}+T\right)
$$

and

$$
\left\{\begin{array}{l}
z^{\prime}(t)=\Phi(z(t)) \quad \text { for all } t \in\left(t_{1}, t_{1}+T\right) \\
z\left(t_{1}\right)=c
\end{array}\right.
$$

Then

$$
y(t) \geq z(t) \quad \text { for all } t \in\left(t_{1}, t_{1}+T\right) .
$$

Proof. For $\eta>0$ we let $z_{\eta} \in C^{1}\left(\left[t_{1}, t_{1}+T_{\eta}\right)\right)$ denote the maximally extended solution of

$$
\left\{\begin{array}{l}
z_{\eta}^{\prime}(t)=\Phi\left(z_{\eta}(t)\right), \quad t \in\left(t_{1}, t_{1}+T_{\eta}\right), \\
z_{\eta}\left(t_{1}\right)=c-\eta .
\end{array}\right.
$$

Then by a continuous dependence argument we have $\lim \sup _{\eta \searrow 0} T_{\eta} \geq T$, and hence for proving (2.2) it is sufficient to show that $y(t)>z_{\eta}(t)$ for all $t \in\left[t_{1}, t_{1}+\hat{T}_{\eta}\right)$ with $\hat{T}_{\eta}:=\min \left\{T, T_{\eta}\right\}$. Indeed, if this was false then there would exist $t_{2} \in\left(t_{1}, t_{1}+\hat{T}_{\eta}\right)$ such that $y>z_{\eta}$ on $\left[t_{1}, t_{2}\right)$ and

$$
y\left(t_{2}\right)=z_{\eta}\left(t_{2}\right) .
$$


Integrating (2.3) and using the monotonicity of $\Phi$ along with (2.1) we then would obtain

$$
\begin{aligned}
z_{\eta}\left(t_{2}\right) & =c-\eta+\int_{t_{1}}^{t_{2}} \Phi\left(z_{\eta}(\bar{t})\right) d \bar{t} \leq c-\eta+\int_{t_{1}}^{t_{2}} \Phi(y(\bar{t})) d \bar{t} \\
& <c+\int_{t_{1}}^{t_{2}} \Phi(y(\bar{t})) d \bar{t} \leq y\left(t_{2}\right),
\end{aligned}
$$

which contradicts (2.4) and ends the proof.

Next, by explicit construction we provide a family of time-independent functions that will essentially play the role of test functions for (1.2) in the proof of Lemma 2.3 below:

Lemma 2.2. Let $F_{0}>0$ and let $\delta \in(0,1)$ be such that $\delta>\frac{2-F_{0}}{2}$. Then there exist positive constants $a, b, \xi, k_{0}$ and $K_{0}$ such that for any choice of $\gamma>0$ the function $\varphi=\varphi^{(\gamma)}:(0, \infty) \rightarrow(0, \infty)$ defined by

$$
\varphi(s):= \begin{cases}\frac{a}{\gamma^{\delta}} s^{-\delta}-b & \text { if } 0<s<\frac{\xi}{\gamma}, \\ e^{-\gamma s} & \text { if } s \geq \frac{\xi}{\gamma}\end{cases}
$$

belongs to $W_{\mathrm{loc}}^{2, \infty}((0, \infty))$ and satisfies

$$
4 s \varphi_{s s}+\left(8-2 F_{0}\right) \varphi_{s} \geq k_{0} \gamma \varphi \quad \text { a.e.in }(0, \infty)
$$

as well as

$$
\int_{0}^{\infty} \frac{\varphi^{2}(s)}{\left|\varphi_{s}(s)\right|} d s \leq \frac{K_{0}}{\gamma^{2}}
$$

Proof. Given $F_{0}$ and $\delta$, we fix $c_{1}>0$ large enough fulfilling $c_{1}>F_{0}+2 \delta-4$, so that

$$
\xi:=\frac{c_{1}+4-F_{0}}{2}
$$

satisfies $\xi>\delta$. Therefore

$$
a:=\frac{\xi^{\delta+1}}{\delta} e^{-\xi} \quad \text { and } \quad b:=\left(\frac{\xi}{\delta}-1\right) e^{-\xi}
$$

are both positive. Now defining $\varphi$ as in (2.5), we have

$$
\lim _{s \nearrow \frac{\xi}{\gamma}} \varphi(s)=\frac{a}{\gamma^{\delta}} \cdot\left(\frac{\xi}{\gamma}\right)^{-\delta}-b=\frac{\xi^{\delta+1}}{\delta} e^{-\xi} \cdot \xi^{-\delta}-\left(\frac{\xi}{\delta}-1\right) e^{-\xi}=e^{-\xi}
$$


and hence $\varphi$ is continuous on $(0, \infty)$. Since moreover

$$
\varphi_{s}(s)= \begin{cases}-\frac{a \delta}{\gamma^{\delta}} s^{-\delta-1} & \text { if } 0<s<\frac{\xi}{\delta}, \\ -\gamma e^{-\gamma s} & \text { if } s>\frac{\xi}{\gamma},\end{cases}
$$

we find

$$
\lim _{s \nearrow \frac{\xi}{\gamma}} \varphi_{S}(s)=-\frac{a \delta}{\gamma^{\delta}} \cdot\left(\frac{\xi}{\gamma}\right)^{-\delta-1}=-\gamma \xi^{\delta+1} e^{-\xi} \cdot \xi^{-\delta-1}=-\gamma e^{-\xi}
$$

and we infer that also $\varphi_{s}$ is continuous on $(0, \infty)$ and therefore clearly $\varphi \in$ $W_{\text {loc }}^{2, \infty}((0, \infty))$. A further differentiation of (2.8) shows that for large $s$

$$
\begin{aligned}
4 s \varphi_{s s}+\left(8-2 F_{0}\right) \varphi_{s} & =4 \gamma^{2} s e^{-\gamma s}-\left(8-2 F_{0}\right) \gamma e^{-\gamma s} \\
& =\left[4 \gamma s-8+2 F_{0}\right] \gamma e^{-\gamma s} \\
& \geq\left[4 \xi-8+2 F_{0}\right] \gamma e^{-\gamma s} \\
& =2 c_{1} \gamma \varphi(s) \quad \text { for all } s>\frac{\xi}{\gamma}
\end{aligned}
$$

whereas for small $s$

$$
\begin{aligned}
4 s \varphi_{s s}+\left(8-2 F_{0}\right) \varphi_{s} & =\frac{4 a \delta(\delta+1)}{\gamma^{\delta}} s^{-\delta-1}-\frac{\left(8-2 F_{0}\right) a \delta}{\gamma^{\delta}} s^{-\delta-1} \\
& =\frac{4 a \delta}{\gamma^{\delta}}\left(\delta-\frac{2-F_{0}}{2}\right) s^{-\delta-1} \\
& \geq \frac{4 a \delta}{\gamma^{\delta}}\left(\delta-\frac{2-F_{0}}{2}\right) \cdot \frac{\gamma}{\xi} s^{-\delta} \quad \text { for all } s<\frac{\xi}{\gamma}
\end{aligned}
$$

because $\delta>\frac{2-F_{0}}{2}$. Since $b>0$, on the other hand we have $\varphi(s) \leq \frac{a}{\gamma^{\delta}} s^{-\delta}$ and therefore

$$
4 s \varphi_{s s}+\left(8-2 F_{0}\right) \varphi_{s} \geq \frac{4 \delta\left(\delta-\frac{2-F_{0}}{2}\right)}{\xi} \cdot \gamma \cdot \varphi(s) \quad \text { for all } s<\frac{\xi}{\gamma} .
$$

Combined with (2.9), this establishes (2.6) upon an obvious choice of $k_{0}$, so that it remains to prove (2.7) for suitably large $K_{0}$. To this end, we compute

$$
\int_{\frac{\xi}{\gamma}}^{\infty} \frac{\varphi^{2}(s)}{\left|\varphi_{s}(s)\right|} d s=\frac{1}{\gamma} \int_{\frac{\xi}{\gamma}}^{\infty} e^{-\gamma s} d s=\frac{1}{\gamma^{2}} e^{-\xi}
$$


and estimate, again using the positivity of $b$,

$$
\int_{0}^{\frac{\xi}{\gamma}} \frac{\varphi^{2}(s)}{\left|\varphi_{s}(s)\right|} d s<\int_{0}^{\frac{\xi}{\gamma}} \frac{\frac{a^{2}}{\gamma^{2 \delta}} s^{-2 \delta}}{\frac{a \delta}{\gamma^{\delta}} s^{-\delta-1}} d s=\frac{a \xi^{2-\delta}}{\delta(2-\delta) \gamma^{2}}
$$

Evidently, (2.7) is a consequence of (2.10) and (2.11).

We are now in the position to state our key result in respect of immediate blowup:

Lemma 2.3. Suppose that $F_{0}>0$ and that $W_{0}$ satisfies (1.3) for some $\mu>0$. Then for any positive $\alpha>\frac{2-F_{0}}{2}$ and each $t_{0} \geq 0$ the proper solution $W$ of (1.2) satisfies

$$
\sup _{s>0, t \in\left(t_{0}, t_{0}+\tau\right)} \frac{W(s, t)}{s^{\alpha}}=\infty \quad \text { for all } \tau>0 .
$$

In particular, we have

$$
\left\|W_{S}\right\|_{L^{\infty}\left((0, \infty) \times\left(t_{0}, t_{0}+\tau\right)\right)}=\infty \quad \text { for all } \tau>0 .
$$

Proof. If the lemma were false, there would exist $\alpha>\frac{2-F_{0}}{2}, t_{0} \geq 0$ and $c_{1}>0$ such that

$$
W(s, t) \leq c_{1} s^{\alpha} \quad \text { for all } s \geq 0 \text { and } t \in\left[t_{0}, t_{0}+\tau\right] .
$$

Our goal is to show that then there exists some large $\gamma$ such that, with $\varphi=\varphi^{(\gamma)}$ taken from Lemma 2.2, the function

$$
y(t):=\int_{0}^{\infty} \varphi(s) W(s, t) d s, \quad t>0,
$$

blows up before $t=t_{0}+\tau$. To this end, we note that since $\alpha>\frac{2-F_{0}}{2}$ and $F_{0}>0$, we can fix $\delta \in(0,1)$ such that

$$
\delta<\alpha \quad \text { and } \quad \delta \geq \frac{2-F_{0}}{2},
$$

and then let the positive constants $a, b, \xi, k_{0}$ and $K_{0}$ be as provided by Lemma 2.2. We next pick $\kappa>0$ small fulfilling

$$
\kappa \leq \frac{k_{0} \tau}{8}
$$

so that with $t_{1}:=t_{0}+\frac{\tau}{2}$, the number

$$
c_{2}:=W\left(\kappa, t_{1}\right)
$$


is positive. We here use the fact that $W$ is positive in $\left(\frac{\kappa}{2}, \infty\right) \times(0, \infty)$ by the strong maximum principle applied to (1.2), which in fact is uniformly parabolic in $\left(\frac{\kappa}{2}, \infty\right) \times(0, \infty)$. Finally, we let $\gamma>1$ be large enough fulfilling

$$
\gamma>\frac{\xi}{\kappa}
$$

and

$$
1+\frac{k_{0} K_{0}}{c_{2}} \cdot e^{\kappa \gamma} \leq e^{2 \kappa \gamma} .
$$

Upon these choices, we let $\varphi:=\varphi^{(\gamma)}$ be as given by $(2.5)$ and, for $\varepsilon \in(0,1)$, we multiply the PDE in $(1.8)$ by $\varphi(s) \chi^{(\varepsilon)}(s)$. For arbitrary

$$
s_{0}>\left\{\frac{\xi}{\gamma}, \frac{1}{\gamma}\right\},
$$

an integration by parts over $s \in\left(0, s_{0}\right)$ yields

$$
\begin{aligned}
& \frac{d}{d t} \int_{0}^{s_{0}} \varphi(s) \chi^{(\varepsilon)}(s) W^{(\varepsilon)}(s, t) d s \\
= & \int_{0}^{s_{0}} \varphi \chi^{(\varepsilon)} \cdot\left\{4 s W_{s s}^{(\varepsilon)}+\frac{1}{2} \chi^{(\varepsilon)}\left(\left(W^{(\varepsilon)}\right)^{2}\right)_{s}+2 F_{0} \chi^{(\varepsilon)} W_{s}^{(\varepsilon)}\right\} \\
= & 4 \int_{0}^{s_{0}}\left(s \varphi \chi^{(\varepsilon)}\right)_{s s} W^{(\varepsilon)}-\frac{1}{2} \int_{0}^{s_{0}}\left(\varphi\left(\chi^{(\varepsilon)}\right)^{2}\right)_{s}\left(W^{(\varepsilon)}\right)^{2} \\
& -2 F_{0} \int_{0}^{s_{0}}\left(\varphi\left(\chi^{(\varepsilon)}\right)^{2}\right)_{s} W^{(\varepsilon)}+B(t)
\end{aligned}
$$

with

$$
\begin{aligned}
B(t):=\left\{4 s \varphi \chi^{(\varepsilon)} W_{s}^{(\varepsilon)}-4\left(s \varphi \chi^{(\varepsilon)}\right)_{s} W^{(\varepsilon)}\right. & +\frac{1}{2} \varphi\left(\chi^{(\varepsilon)}\right)^{2}\left(W^{(\varepsilon)}\right)^{2} \\
& \left.+2 F_{0} \varphi\left(\chi^{(\varepsilon)}\right)^{2} W^{(\varepsilon)}\right\}\left.\right|_{0} ^{s_{0}}
\end{aligned}
$$

for $t>0$. Here we calculate

$$
\left(s \varphi \chi^{(\varepsilon)}\right)_{s}=s \varphi_{s} \chi^{(\varepsilon)}+\varphi \chi^{(\varepsilon)}+s \varphi \chi_{s}^{(\varepsilon)}
$$

and

$$
\left(s \varphi \chi^{(\varepsilon)}\right)_{s s}=s \varphi_{s s} \chi^{(\varepsilon)}+2 \varphi_{s} \chi^{(\varepsilon)}+2 s \varphi_{s} \chi_{s}^{(\varepsilon)}+2 \varphi \chi_{s}^{(\varepsilon)}+s \varphi \chi_{s s}^{(\varepsilon)}
$$


as well as

$$
\left(\varphi\left(\chi^{(\varepsilon)}\right)^{2}\right)_{s}=\varphi_{s}\left(\chi^{(\varepsilon)}\right)^{2}+2 \varphi \chi^{(\varepsilon)} \chi_{s}^{(\varepsilon)}
$$

Thus, since $\chi^{(\varepsilon)} \equiv 0$ on $\left(0, \frac{\varepsilon}{2}\right)$ and $\chi^{(\varepsilon)} \equiv 1$ on $(\varepsilon, \infty) \ni s_{0}$, the boundary terms in (2.22) become

$$
\begin{aligned}
B(t)= & 4 s_{0} e^{-\gamma s_{0}} W_{s}^{(\varepsilon)}\left(s_{0}, t\right)+4\left[\gamma s_{0} e^{-\gamma s_{0}}-e^{-\gamma s_{0}}\right] \cdot W^{(\varepsilon)}\left(s_{0}, t\right) \\
& +\frac{1}{2} e^{-\gamma s_{0}}\left(W^{(\varepsilon)}\left(s_{0}, t\right)\right)^{2}+2 F_{0} e^{-\gamma s_{0}} W^{(\varepsilon)}\left(s_{0}, t\right) \quad \text { for } t>0 .
\end{aligned}
$$

Recalling that $W_{s}^{(\varepsilon)} \geq 0$, dropping the non-negative terms we thus find that

$$
\begin{aligned}
B(t) & \geq 4\left(\gamma s_{0}-1\right) e^{-\gamma s_{0}} W^{(\varepsilon)}\left(s_{0}, t\right) \\
& \geq 0 \quad \text { for all } t>0
\end{aligned}
$$

due to (2.21). Therefore, using (2.23) and (2.24) in (2.22) we obtain

$$
\begin{aligned}
& \frac{d}{d t} \int_{0}^{s_{0}} \varphi(s) \chi^{(\varepsilon)}(s) W^{(\varepsilon)}(s, t) d s \geq 4 \int_{0}^{s_{0}} s \varphi_{s s} \chi^{(\varepsilon)} W^{(\varepsilon)}+8 \int_{0}^{s_{0}} \varphi_{s} \chi^{(\varepsilon)} W^{(\varepsilon)} \\
& \quad+8 \int_{0}^{s_{0}} s \varphi_{s} \chi_{s}^{(\varepsilon)} W^{(\varepsilon)}+8 \int_{0}^{s_{0}} \varphi \chi_{s}^{(\varepsilon)} W^{(\varepsilon)}+4 \int_{0}^{s_{0}} s \varphi \chi_{s s}^{(\varepsilon)} W^{(\varepsilon)} \\
& \quad-\frac{1}{2} \int_{0}^{s_{0}} \varphi_{s}\left(\chi^{(\varepsilon)}\right)^{2}\left(W^{(\varepsilon)}\right)^{2}-\int_{0}^{s_{0}} \varphi \chi^{(\varepsilon)} \chi_{s}^{(\varepsilon)}\left(W^{(\varepsilon)}\right)^{2} \\
& \quad-2 F_{0} \int_{0}^{s_{0}} \varphi_{s}\left(\chi^{(\varepsilon)}\right)^{2} W^{(\varepsilon)}-4 F_{0} \int_{0}^{s_{0}} \varphi \chi^{(\varepsilon)} \chi_{s}^{(\varepsilon)} W^{(\varepsilon)} \\
& =\int_{0}^{s_{0}}\left\{4 s \varphi_{s s}+8 \varphi_{s}-2 F_{0} \varphi_{s} \chi^{(\varepsilon)}\right\} \cdot \chi^{(\varepsilon)} W^{(\varepsilon)} \\
& \quad-\frac{1}{2} \int_{0}^{s_{0}} \varphi_{s}\left(\chi^{(\varepsilon)}\right)^{2}\left(W^{(\varepsilon)}\right)^{2}+I_{1}(t)+I_{2}(t) \quad \text { for all } t>0
\end{aligned}
$$

with

$$
I_{1}(t):=\int_{0}^{s_{0}}\left\{8 s \varphi_{s}+8 \varphi-\varphi \chi^{(\varepsilon)} W^{(\varepsilon)}-4 F_{0} \varphi \chi^{(\varepsilon)}\right\} \cdot \chi_{s}^{(\varepsilon)} W^{(\varepsilon)}
$$

and

$$
I_{2}(t):=4 \int_{0}^{s_{0}} s \varphi \chi_{s s}^{(\varepsilon)} W^{(\varepsilon)}
$$


Now using that $\chi_{s}^{(\varepsilon)} \leq \frac{c_{\chi}}{\varepsilon},\left|\chi_{s s}^{(\varepsilon)}\right| \leq \frac{c_{\chi}}{\varepsilon^{2}}$ and $\chi_{s}^{(\varepsilon)} \equiv 0$ on $(\varepsilon, \infty)$, in view of our hypothesis (2.14) on $W$ and the facts that $W^{(\varepsilon)} \leq W \leq \frac{\mu}{\pi}$ and $\chi^{(\varepsilon)} \leq 1$, we can estimate

$$
\begin{aligned}
I_{1}(t) & \geq-\int_{0}^{\varepsilon}\left\{8 s\left|\varphi_{s}\right|+\frac{\mu}{\pi} \varphi+4 F_{0} \varphi\right\} \cdot \frac{c_{\chi}}{\varepsilon} \cdot c_{1} s^{\alpha} d s \\
& \geq-\frac{c_{\chi} c_{1}}{\varepsilon} \cdot \frac{\left[8 \delta+\frac{\mu}{\pi}+4 F_{0}\right] a}{\gamma^{\delta}} \cdot \int_{0}^{\varepsilon} s^{-\delta+\alpha} d s \quad \text { for all } t \in\left(t_{0}, t_{0}+\tau\right)
\end{aligned}
$$

and similarly

$$
\left|I_{2}(t)\right| \leq \frac{4 c_{\chi} c_{1}}{\varepsilon^{2}} \cdot \frac{a}{\gamma^{\delta}} \cdot \int_{0}^{\varepsilon} s^{1-\delta+\alpha} d s \quad \text { for all } t \in\left(t_{0}, t_{0}+\tau\right)
$$

whenever $\varepsilon<\frac{\xi}{\gamma}$, because in that case we know that $0 \leq \varphi(s) \leq \frac{a}{\gamma^{\delta}} s^{-\delta}$ for all $s \in(0, \varepsilon)$. Since $\delta<\alpha$, we conclude that there exists $c_{3}>0$ such that

$$
I_{1}(t)+I_{2}(t) \geq-c_{3} \varepsilon^{\alpha-\delta} \quad \text { for all } t \in\left(t_{0}, t_{0}+\tau\right) .
$$

Next, the property (2.6) of $\varphi$ implies that

$$
4 s \varphi_{s s}+8 \varphi_{s}-2 F_{0} \varphi_{s} \chi^{(\varepsilon)} \geq k_{0} \gamma \varphi+2 F_{0} \varphi_{s}\left(1-\chi^{(\varepsilon)}\right) \quad \text { a.e. in }(0, \infty),
$$

so that altogether an integration of (2.25) shows that

$$
y^{\left(\varepsilon, s_{0}\right)}(t):=\int_{0}^{s_{0}} \varphi(s) \chi^{(\varepsilon)}(s) W^{(\varepsilon)}(s, t) d s, t \geq 0,
$$

satisfies

$$
\begin{aligned}
y^{\left(\varepsilon, s_{0}\right)}(t) \geq & y^{\left(\varepsilon, s_{0}\right)}\left(t_{1}\right)+k_{0} \gamma \int_{t_{1}}^{t} \int_{0}^{s_{0}} \varphi \chi^{(\varepsilon)} W^{(\varepsilon)} \\
& +2 F_{0} \int_{t_{1}}^{t} \int_{0}^{s_{0}} \varphi_{s}\left(1-\chi^{(\varepsilon)}\right) \chi^{(\varepsilon)} W^{(\varepsilon)} \\
& -\frac{1}{2} \int_{t_{1}}^{t} \int_{0}^{s_{0}} \varphi_{s}\left(\chi^{(\varepsilon)}\right)^{2}\left(W^{(\varepsilon)}\right)^{2}-c_{3} \varepsilon^{\alpha-\delta} \cdot\left(t-t_{1}\right) \quad \text { for all } t \in\left(t_{1}, t_{0}+\tau\right),
\end{aligned}
$$

provided that $\varepsilon<\frac{\xi}{\gamma}$. Invoking the dominated convergence theorem along with the exponential decay of $\varphi(s)$ and $\varphi_{s}(s)$ as $s \rightarrow \infty$, we may take $s_{0} \nearrow \infty$ to see that $y^{(\varepsilon)}(t):=\int_{0}^{\infty} \varphi(s) \chi^{(\varepsilon)}(s) W^{(\varepsilon)}(s, t) d s, t \geq 0$, fulfills

$$
\begin{aligned}
y^{(\varepsilon)}(t) \geq & y^{(\varepsilon)}\left(t_{1}\right)+k_{0} \gamma \int_{t_{1}}^{t} \int_{0}^{\infty} \varphi \chi^{(\varepsilon)} W^{(\varepsilon)}+2 F_{0} \int_{t_{1}}^{t} \int_{0}^{\infty} \varphi_{s}\left(1-\chi^{(\varepsilon)}\right) \chi^{(\varepsilon)} W^{(\varepsilon)} \\
& -\frac{1}{2} \int_{t_{1}}^{t} \int_{0}^{\infty} \varphi_{s}\left(\chi^{(\varepsilon)}\right)^{2}\left(W^{(\varepsilon)}\right)^{2}-c_{3} \varepsilon^{\alpha-\delta} \cdot\left(t-t_{1}\right) \text { for all } t \in\left(t_{1}, t_{0}+\tau\right) .
\end{aligned}
$$


Here, since for some $c_{4}>0$ we have $\left|\varphi_{s}(s)\left(1-\chi^{(\varepsilon)}(s)\right) \chi^{(\varepsilon)}(s) W^{(\varepsilon)}(s, t)\right| \leq\left|\varphi_{s}(s) W(s, t)\right| \leq c_{4}\left(1+s^{\alpha-\delta-1}\right) \cdot e^{-\gamma s}$ for all $s>0$ and $t \in\left[t_{0}, t_{0}+\tau\right]$, by (2.14) and the definition of $\varphi$, one more application of the dominated convergence theorem asserts that

$$
2 F_{0} \int_{t_{1}}^{t} \int_{0}^{\infty} \varphi_{s}\left(1-\chi^{(\varepsilon)}\right) \chi^{(\varepsilon)} W^{(\varepsilon)} \rightarrow 0 \quad \text { as } \varepsilon \searrow 0
$$

for all $t \in\left(t_{1}, t_{0}+\tau\right)$, because $\delta<\alpha$. In the remaining terms making up (2.27), we apply the monotone convergence theorem in taking $\varepsilon \searrow 0$ to conclude that again since $\alpha>\delta$, the function $y$ defined by (2.15) satisfies

$$
y(t) \geq y\left(t_{1}\right)+k_{0} \gamma \int_{t_{1}}^{t} \int_{0}^{\infty} \varphi W-\frac{1}{2} \int_{t_{1}}^{t} \int_{0}^{\infty} \varphi_{s} W^{2} \quad \text { for all } t \in\left(t_{1}, t_{0}+\tau\right),
$$

because $\chi^{(\varepsilon)} \nearrow 1$ on $(0, \infty)$ and $W^{(\varepsilon)} \nearrow W$ in $(0, \infty) \times(0, \infty)$. In order to take advantage of the quadratic term in (2.28), we employ Hölder's inequality and (2.7) to see that

$$
\begin{aligned}
y^{2}(t) & =\left(\int_{0}^{\infty} \varphi W\right)^{2} \leq\left(\int_{0}^{\infty} \frac{\varphi^{2}}{\left|\varphi_{s}\right|}\right) \cdot\left(\int_{0}^{\infty}\left|\varphi_{s}\right| W^{2}\right) \\
& \leq \frac{K_{0}}{\gamma^{2}} \cdot \int_{0}^{\infty}\left|\varphi_{s}\right| W^{2} \quad \text { for all } t>0,
\end{aligned}
$$

and thereby (2.28) becomes

$$
y(t) \geq y\left(t_{1}\right)+k_{0} \gamma \int_{t_{1}}^{t} y(\bar{t}) d \bar{t}+\frac{\gamma^{2}}{K_{0}} \cdot \int_{t_{1}}^{t} y^{2}(\bar{t}) d \bar{t} \quad \text { for all } t \in\left(t_{1}, t_{0}+\tau\right) .
$$

Consequently, Lemma 2.1 states that

$$
y(t) \geq z(t) \quad \text { for all } t \in\left(t_{1}, t_{0}+\tau\right),
$$

where $z$ is the solution of

$$
\left\{\begin{array}{l}
z^{\prime}=A z+B z^{2}, \quad t>t_{1} \\
z\left(t_{1}\right)=y\left(t_{1}\right)
\end{array}\right.
$$

with

$$
A:=k_{0} \gamma \quad \text { and } \quad B:=\frac{\gamma^{2}}{K_{0}}
$$


The solution of this Bernoulli-type initial-value problem is explicitly given by

$$
z(t)=\frac{1}{\left(\frac{1}{y\left(t_{1}\right)}+\frac{B}{A}\right) e^{-A\left(t-t_{1}\right)}-\frac{B}{A}}, \quad t \in\left(t_{1}, t_{1}+T\right),
$$

with its maximum existence time determined by

$$
T=\frac{1}{A} \ln \left(1+\frac{A}{B y\left(t_{1}\right)}\right) \text {. }
$$

Now by (2.18) and the fact that $W_{s} \geq 0$, we can estimate $y\left(t_{1}\right)$ from below according to

$$
y\left(t_{1}\right)=\int_{0}^{\infty} \varphi(s) W\left(s, t_{1}\right) d s \geq c_{2} \int_{\kappa}^{\infty} \varphi(s) d s=c_{2} \int_{\kappa}^{\infty} e^{-\gamma s} d s=\frac{c_{2}}{\gamma} e^{-\kappa \gamma},
$$

because (2.19) ensures that $\kappa>\frac{\xi}{\gamma}$ and hence $\varphi(s)=e^{-\gamma s}$ for all $s>\kappa$. Thus, by (2.30) and (2.20) we have

$$
\begin{aligned}
T & \leq \frac{1}{k_{0} \gamma} \cdot \ln \left(1+\frac{k_{0} \gamma}{\frac{\gamma^{2}}{K_{0}} \cdot \frac{c_{2}}{\gamma} \cdot e^{-\kappa \gamma}}\right)=\frac{1}{k_{0} \gamma} \cdot \ln \left(1+\frac{k_{0} K_{0}}{c_{2}} e^{\kappa \gamma}\right) \\
& \leq \frac{1}{k_{0} \gamma} \cdot \ln \left(e^{2 \kappa \gamma}\right)=\frac{2 \kappa}{k_{0}} .
\end{aligned}
$$

But in light of (2.17) this means that $T \leq \frac{\tau}{4}$, so that (2.29) and (2.31) entail that $y$ blows up before or at $t=t_{1}+\frac{\tau}{4}=t_{0}+\frac{3 \tau}{4}$. Since $\delta<1$ and hence $\varphi$ is integrable over $(0, \infty)$, this is evidently incompatible with the boundedness of $W$ and thereby proves that the assumption (2.14) must have been false.

Proof of Theorem 0.1 . The conclusion results upon a reformulation of Lemma 2.3 in terms of $u$ rather than $W$.

\section{Formation of Dirac-type singularities when $\mu>8 \pi-2 f_{0}$}

In this section we shall see that if $\mu>8 \pi-2 f_{0}$ then $u$ will asymptotically exhibit a Dirac-type singularity at the origin. As a first step towards this, let us explicitly construct initial data such that the corresponding solution of (1.2) will lie below $W$, but will be increasing with time. 
Lemma 3.1. Suppose that $F_{0} \geq 0$, and that (1.3) is satisfied with some

$$
\mu>8 \pi-4 \pi F_{0}
$$

Then for all positive $\hat{\mu} \in\left(8 \pi-4 \pi F_{0}, \mu\right)$ there exist $s_{0}>0$ and $\underline{W}_{0} \in W^{1, \infty}((0, \infty)) \cap$ $C^{2}\left([0, \infty) \backslash\left\{s_{0}\right\}\right)$ such that $\underline{W}_{0} \equiv 0$ in $\left[0, s_{0}\right], \liminf _{s \backslash s_{0}} \underline{W}_{0 s}(s)>0, \underline{W}_{0}(s) \rightarrow \frac{\hat{\mu}}{\pi}$ as $s \rightarrow \infty$, and such that

$$
4 s \underline{W}_{0 s s}+\underline{W}_{0} \underline{W}_{0 s}+2 F_{0} \underline{W}_{0 s}=0 \quad \text { in }\left(s_{0}, \infty\right)
$$

as well as

$$
\underline{W}_{0} \leq W_{0} \quad \text { in }(0, \infty)
$$

Proof. Abbreviating $a:=\frac{\hat{\mu}}{\pi}$, from (1.3) we know that there exists some large $s_{1}>$ 0 such that

$$
W_{0}(s) \geq a \quad \text { for all } s \geq s_{1} .
$$

Since $a>8-4 F_{0}$ by assumption, both

$$
b:=\frac{1}{2\left(a+2 F_{0}-4\right)}
$$

and

$$
\beta:=\frac{a+2 F_{0}-4}{4}
$$

are positive and moreover $b<\frac{1}{a}$. Therefore we can pick some small $c>0$ such that

$$
s_{0}:=\left(\frac{\frac{1}{a}-b}{c}\right)^{\frac{1}{\beta}} \geq s_{1} .
$$

We now let

$$
\underline{W}_{0}(s):= \begin{cases}0 & \text { if } s \in\left[0, s_{0}\right], \\ a-\frac{1}{b+c s^{\beta}} & \text { if } s>s_{0},\end{cases}
$$

and we see, using (3.7), that $\underline{W}_{0}$ has the claimed regularity properties, whereas (3.4) and (3.7) guarantee that $\underline{W}_{0} \leq W_{0}$ on $[0, \infty)$. Furthermore, computing

$$
\begin{aligned}
\underline{W}_{0 s}(s) & =\frac{\beta c s^{\beta-1}}{\left(b+c s^{\beta}\right)^{2}} \quad \text { and } \\
\underline{W}_{0 s s}(s) & =\frac{\beta(\beta-1) b c s^{\beta-2}-\beta(\beta+1) c^{2} s^{2 \beta-2}}{\left(b+c s^{\beta}\right)^{3}}
\end{aligned}
$$


for $s>s_{0}$, we see that $\liminf _{s \backslash s_{0}} \underline{W}_{0 s}(s)>0$, and that for $s>s_{0}$,

$$
\begin{aligned}
& 4 s \underline{W}_{0 s s}+\underline{W}_{0} \underline{W}_{0 s}+2 F_{0} \underline{W}_{0 s} \\
& \begin{aligned}
&=\frac{1}{\left(b+c s^{\beta}\right)^{3}} \cdot\left\{\beta(\beta-1) b c s^{\beta-1}-4 \beta(\beta+1) c^{2} s^{2 \beta-1}\right. \\
&\left.+\left[a b+a c s^{\beta}-1\right] \cdot \beta c s^{\beta-1}+2 \beta c F_{0} s^{\beta-1} \cdot\left(b+c s^{\beta}\right)\right\} \\
&=\frac{1}{\left(b+c s^{\beta}\right)^{3}} \cdot\left\{\beta c \cdot\left[4(\beta-1) b+a b-1+2 F_{0} b\right] \cdot s^{\beta-1}\right. \\
&\left.+\beta c^{2} \cdot\left[-4(\beta+1)+a+2 F_{0}\right] \cdot s^{2 \beta-1}\right\} .
\end{aligned}
\end{aligned}
$$

According to (3.5) and (3.6), it can be easily checked that

$$
-4(\beta+1)+a+2 F_{0}=0 \quad \text { and } \quad 4(\beta-1) b+a b-1+2 F_{0} b=0,
$$

whereby (3.2) follows and the proof is complete.

The following statement will be useful in identifying possible limits of solutions of (1.2) as $t \rightarrow \infty$ in Lemma 3.3 below. It rules out non-trivial steady states $\psi$ of (1.2) having their 'mass' $\lim _{s \rightarrow \infty} \psi(s)$ larger than $8 \pi-4 \pi F_{0}$.

Lemma 3.2. Let $F_{0} \geq 0$ and assume that $\psi \in C^{2}((0, \infty))$ is a non-negative solution of

$$
0=4 s \psi_{s s}+\psi \psi_{s}+2 F_{0} \psi_{s}, \quad s>0,
$$

with the additional properties $\psi_{s} \geq 0$ on $(0, \infty)$ and

$$
\psi(s) \nearrow \frac{\mu}{\pi} \quad \text { as } s \rightarrow \infty
$$

with some $\mu \geq 0$. Under these assumptions

$$
\mu>8 \pi-4 \pi F_{0}
$$

then

$$
\psi \equiv \frac{\mu}{\pi} \quad \text { in }(0, \infty)
$$

Proof. We may confine ourselves to the case $\mu>0$, in which we substitute $s=e^{-\xi}$ and let

$$
z(\xi):=\psi\left(e^{-\xi}\right), \quad \xi \in \mathbb{R}
$$

Then computing

$$
\psi_{s}=-e^{\xi} z_{\xi} \quad \text { and } \quad \psi_{s s}=e^{2 \xi}\left(z_{\xi \xi}+z_{\xi}\right),
$$


from (3.8) we obtain

$$
\begin{aligned}
0 & =4 e^{-\xi} \cdot e^{2 \xi}(z \xi \xi+z \xi)-z \cdot\left(e^{\xi} z \xi\right)+2 F_{0} \cdot\left(-e^{\xi} z_{\xi}\right) \\
& =e^{\xi} \cdot\left(4 z \xi \xi+4 z \xi-z z \xi-2 F_{0} z \xi\right), \quad \xi \in \mathbb{R},
\end{aligned}
$$

which shows that if we write $\alpha:=\frac{2-F_{0}}{2}$, we have

$$
0=4 z \xi \xi+4 \alpha z \xi-z z \xi=\left(4 z \xi+4 \alpha z-\frac{1}{2} z^{2}\right)_{\xi} \quad \text { on } \mathbb{R} .
$$

Therefore there exists $c \in \mathbb{R}$ such that $4 z \xi+4 \alpha z-\frac{1}{2} z^{2}+4 c \equiv 0$ or, equivalently,

$$
z_{\xi}=-\alpha z+\frac{1}{8} z^{2}-c \quad \text { on } \mathbb{R} .
$$

By (3.9), $c$ is determined by taking $\xi \rightarrow-\infty$ and thus satisfies

$$
c=-\alpha \cdot \frac{\mu}{\pi}+\frac{1}{8} \cdot\left(\frac{\mu}{\pi}\right)^{2}=\frac{\mu}{8 \pi} \cdot\left(-8+4 F_{0}+\frac{\mu}{\pi}\right)>0
$$

according to (3.10). Using that $\psi_{s} \geq 0$, we have $z_{\xi} \leq 0$ on $\mathbb{R}$ and hence an ODE argument applied to (3.12) shows that $z(\xi) \searrow z_{\infty}$ as $\xi \rightarrow+\infty$, where $z_{\infty}$ must be a steady state of (3.12) and thus satisfy

$$
0=-\alpha z_{\infty}+\frac{1}{8} z_{\infty}^{2}-c
$$

that is,

$$
z_{\infty} \in\left\{4 \alpha+\sqrt{16 \alpha^{2}+8 c}, 4 \alpha-\sqrt{16 \alpha^{2}+8 c}\right\}=\left\{\frac{\mu}{\pi}, 4 \alpha-\sqrt{16 \alpha^{2}+8 c}\right\} .
$$

Since $4 \alpha<\sqrt{16 \alpha^{2}+8 c}$ by (3.13), this means that $z_{\infty}=\frac{\mu}{\pi}$ and therefore $z$ must be a constant, as claimed.

The following lemma prepares the main result of this section.

Lemma 3.3. Let $F_{0} \geq 0$ and suppose that (1.3) is fulfilled with some

$$
\mu>8 \pi-4 \pi F_{0} .
$$

Then the proper solution $W$ of (1.2) satisfies

$$
W(s, t) \rightarrow \frac{\mu}{\pi} \quad \text { as } t \rightarrow \infty,
$$

the convergence being uniform on compact subsets of $(0, \infty)$. 
Proof. We let $\delta>0$ be given and fix a positive $\hat{\mu} \in\left(8 \pi-4 \pi F_{0}, \mu\right)$ close enough to $\mu$ such that

$$
\frac{\hat{\mu}}{\pi}>\frac{\mu}{\pi}-\delta
$$

Applying Lemma 3.1 provides a minorant $\underline{W}_{0}$ of $W_{0}$ with the properties listed in that lemma. According to Lemma 1.5 and Lemma 1.6, the corresponding proper solution $\underline{W}$ satisfies $\underline{W} \leq W$ in $[0, \infty) \times[0, \infty)$ and

$$
\underline{W}_{t} \geq 0 \quad \text { in }(0, \infty) \times(0, \infty) \text {. }
$$

Since $W \leq \frac{\mu}{\pi}$ in $[0, \infty) \times[0, \infty)$, in view of (3.16) and the monotonicity of $\underline{W}$ with respect to $s$, it is sufficient for proving (3.15) to show that

$$
\underline{W}(s, t) \nearrow \frac{\hat{\mu}}{\pi} \quad \text { as } t \rightarrow \infty
$$

for all $s>0$. To this end, we note that due to (3.17) we have

$$
\underline{W}(s, t) \nearrow \psi(s) \quad \text { as } t \rightarrow \infty
$$

with some non-negative limit function $\psi$ that clearly is non-decreasing on $(0, \infty)$ and satisfies $\psi \leq \frac{\hat{\mu}}{\pi}$ on $(0, \infty)$ as well as

$$
\psi(s) \nearrow \frac{\hat{\mu}}{\pi} \quad \text { as } s \rightarrow \infty
$$

Since $\underline{W}$ solves the PDE in (1.2) classically in $(0, \infty) \times(0, \infty)$, parabolic estimates ensure that the convergence in (3.19) is locally uniform in $(0, \infty) \times(0, \infty)$, which entails that $\psi$ is smooth in $(0, \infty)$ and solves

$$
0=4 s \psi_{s s}+\psi \psi_{s}+2 F_{0} \psi_{s} \quad \text { in }(0, \infty) .
$$

Along with (3.20) and the fact that $\hat{\mu}>8 \pi-4 \pi F_{0}$, in view of Lemma 3.2 this means that $\psi \equiv \frac{\hat{\mu}}{\pi}$ and thereby establishes (3.18).

Proof of Theorem 0.2 i). The assertion is precisely that of Lemma 3.3, restated in the original variables.

\section{Emergence of mild singularities for $\mu<8 \pi-2 f_{0}$}

In order to complete our identification of the number $\mu=8 \pi-2 f_{0}$ as a critical mass in (0.2), in this section our goal is to show that if the cellular mass is below this threshold then the singularity of the solution will be comparatively mild in the sense that $u(\cdot, t)$ remains bounded in some $L^{p}$ space for all times. To begin with, we establish an upper bound for $W^{(\varepsilon)}$ near $s=0$ that is independent of $t$ and $\varepsilon$ : 
Lemma 4.1. Suppose that $F_{0} \in[0,2)$, and that $W_{0}$ satisfies (1.3) with some

$$
\mu<8 \pi-4 \pi F_{0} .
$$

Then there exists $C>0$ such that for all $\varepsilon>0$ the solution of (1.8) fulfills

$$
W^{(\varepsilon)}(s, t) \leq C s^{\frac{2-F_{0}}{2}} \quad \text { for all } s \geq 0 \text { and } t \geq 0 .
$$

Proof. Writing $\alpha:=\frac{2-F_{0}}{2}$, for $a>0$ we introduce

$$
\psi^{(a)}(s):=\frac{a s^{\alpha}}{1+\frac{a}{8 \alpha} s^{\alpha}}, \quad s \geq 0,
$$

and compute

$$
\psi_{s}^{(a)}(s)=\frac{\alpha a s^{\alpha-1}}{\left(1+\frac{a}{8 \alpha} s^{\alpha}\right)^{2}}
$$

and

$$
\psi_{s s}^{(a)}(s)=-\frac{\alpha(1-\alpha) a s^{\alpha-2}+\frac{1}{8}(\alpha+1) a^{2} s^{2 \alpha-2}}{\left(1+\frac{a}{8 \alpha} s^{\alpha}\right)^{3}}
$$

for $s>0$. We observe that $\psi^{(a)}(s)$ increases both with $s$ and with $a$, and that

$$
\psi^{(a)}(s) \nearrow 8 \alpha \quad \text { as } a \rightarrow \infty
$$

for all $s>0$. Since $W_{0}(s) \leq \frac{\mu}{\pi}<8 \alpha$ for all $s \geq 0$ by (4.1), and since

$$
\liminf _{s \searrow 0} \psi_{s}^{(a)}(s) \rightarrow+\infty \text { as } a \rightarrow \infty,
$$

from the fact that $W_{0 s} \in L_{\text {loc }}^{\infty}([0, \infty))$ we easily infer that for some large $a>0$ we have

$$
W_{0}(s) \leq \psi^{(a)}(s) \quad \text { for all } s \geq 0 .
$$

Now by (4.4) and (4.5), we find that

$$
\begin{aligned}
& 4 s \psi_{s s}^{(a)}+\psi^{(a)} \psi_{s}^{(a)}+2 F_{0} \psi_{s}^{(a)}= \frac{-4 \alpha(1-\alpha) a s^{\alpha-1}-\frac{1}{2}(\alpha+1) a^{2} s^{2 \alpha-1}}{\left(1+\frac{a}{8 \alpha} s^{\alpha}\right)^{3}} \\
&+\frac{a s^{\alpha} \cdot \alpha a s^{\alpha-1}}{\left(1+\frac{a}{8 \alpha} s^{\alpha}\right)^{3}}+\frac{2 \alpha a F_{0} s^{\alpha-1}+\frac{1}{4} a^{2} F_{0} s^{2 \alpha-1}}{\left(1+\frac{a}{8 \alpha} s^{\alpha}\right)^{3}} \\
&= \frac{1}{\left(1+\frac{a}{8 \alpha} s^{\alpha}\right)^{3}} \cdot\left\{\alpha a\left[-4(1-\alpha)+2 F_{0}\right] s^{\alpha-1}\right. \\
&=0 \quad \text { for all } s>0
\end{aligned}
$$


because of the definition of $\alpha$. Since $0 \leq \chi^{(\varepsilon)} \leq 1$ and $\psi_{s}^{(a)} \geq 0$, this entails that

$$
-4 s \psi_{s s}^{(a)}-\chi^{(\varepsilon)} \psi^{(a)} \psi_{s}^{(a)}-2 F_{0} \chi^{(\varepsilon)} \psi_{s}^{(a)} \geq 0 \quad \text { in }(0, \infty)
$$

and hence $\psi^{(a)}$ is a stationary supersolution of (1.8). Therefore in view of (4.6), by comparison we infer that $W^{(\varepsilon, S)}(s, t) \leq \psi^{(a)}(s)$ and hence

$$
W^{(\varepsilon)}(s, t) \leq \psi^{(a)}(s) \quad \text { for all } s \geq 0 \text { and } t \geq 0 .
$$

Using (4.3), from this we immediately obtain (4.2).

Using a Bernstein-type technique, from the above result we can derive the natural analogue for the spatial derivative of $W$ :

Lemma 4.2. Let $F_{0} \in[0,2)$ and suppose that (1.3) is satisfied with some $\mu<$ $8 \pi-4 \pi F_{0}$. Then for all $\tau>0$ there exists $C(\tau)>0$ such that the proper solution W of (1.2) fulfills

$$
W_{s}(s, t) \leq C(\tau)\left(1+s^{-\frac{F_{0}}{2}}\right) \quad \text { for all } s>0 \text { and any } t>\tau .
$$

Proof. Since in $\left(\frac{1}{2}, \infty\right) \times(0, \infty)$ we know that $W$ is a bounded smooth solution of the uniformly parabolic PDE in (1.2), interior parabolic regularity theory ensures that $W_{s}$ is bounded in $(1, \infty) \times(\tau, \infty)$, and hence we only need to establish (4.7) for $s \in(0,1]$ and $t>\tau$. For this purpose, we fix $\varepsilon \in(0,1)$ and let the non-negative function $Q=Q(s, t)$ be defined through the substitution

$$
W^{(\varepsilon)}(s, t)=Q^{2}(s, t), \quad s \geq 0, t \geq 0 .
$$

Then, using (1.8), we compute

$$
2 Q Q_{t}=4 s\left(2 Q Q_{s s}+2 Q_{s}^{2}\right)+2 \chi^{(\varepsilon)} Q^{3} Q_{s}+4 F_{0} \chi^{(\varepsilon)} Q Q_{s}
$$

and thus we have

$$
Q_{t}=4 s Q_{s s}+4 s \frac{Q_{s}^{2}}{Q}+\chi^{(\varepsilon)} Q^{2} Q_{s}+2 F_{0} \chi^{(\varepsilon)} Q_{s} \quad \text { for } s>0 \text { and } t>0 .
$$

By further differentiation,

$$
\begin{aligned}
Q_{s t}= & 4 s Q_{s s s}+4 Q_{s s}+8 s \frac{Q_{s} Q_{s s}}{Q}-4 s \frac{Q_{s}^{3}}{Q^{2}}+4 \frac{Q_{s}^{2}}{Q}+\chi^{(\varepsilon)} Q^{2} Q_{s s} \\
& +2 \chi^{(\varepsilon)} Q Q_{s}^{2}+\chi_{s}^{(\varepsilon)} Q^{2} Q_{s}+2 F_{0} \chi^{(\varepsilon)} Q_{s s}+2 F_{0} \chi_{s}^{(\varepsilon)} Q_{s} \\
& \quad \text { for } s>0 \text { and } t>0 .
\end{aligned}
$$

We now fix $z \in C^{\infty}([0, \infty))$ such that $z(0)=0, z \equiv 1$ on $[\tau, \infty)$ and $0 \leq z^{\prime}(t) \leq \frac{2}{\tau}$ for all $t \geq 0$, and let

$$
\zeta(s):=\left(s(2-s)^{2}\right)^{\delta}, \quad s \in[0,2]
$$


with

$$
\delta:=\frac{F_{0}+2}{2}
$$

Then

$$
g(s, t):=z(t) \zeta(s) Q_{s}^{2}(s, t), \quad s \in[0,2], t \geq 0,
$$

vanishes at $s=0$, at $s=2$ and whenever $t=0$, so that if for some $T>0, g$ attains a positive maximum over $[0,2] \times[0, T]$ at some point $\left(s_{0}, t_{0}\right) \in[0,2] \times[0, T]$, then necessarily $s_{0} \in(0,2)$ and $t_{0} \in(0, T]$, and hence $g_{s}=0, g_{s s} \leq 0$ and $g_{t} \geq 0$ hold at this point. Since

$$
\begin{aligned}
g_{s} & =2 z \zeta Q_{s} Q_{s s}+z \zeta_{s} Q_{s}^{2} \quad \text { and } \\
g_{s s} & =2 z \zeta Q_{s} Q_{s s s}+2 z \zeta Q_{s s}^{2}+4 z \zeta_{s} Q_{s} Q_{s s}+z \zeta_{s s} Q_{s}^{2},
\end{aligned}
$$

at $\left(s_{0}, t_{0}\right)$ we thus have $Q_{s s}=-\frac{\zeta_{s} Q_{s}}{2 \zeta}$ and therefore by (4.8)

$$
\begin{aligned}
& 0 \leq g_{t}=z^{\prime} \zeta Q_{s}^{2}+2 z \zeta Q_{s} \cdot\left\{4 s Q_{s s s}+4 Q_{s s}+8 s \frac{Q_{s} Q_{s s}}{Q}-4 s \frac{Q_{s}^{3}}{Q^{2}}+4 \frac{Q_{s}^{2}}{Q}\right. \\
& +\chi^{(\varepsilon)} Q^{2} Q_{s s}+2 \chi^{(\varepsilon)} Q Q_{s}^{2}+\chi_{s}^{(\varepsilon)} Q^{2} Q_{s}+2 F_{0} \chi^{(\varepsilon)} Q_{s s} \\
& \left.+2 F_{0} \chi_{s}^{(\varepsilon)} Q_{s}\right\} \\
& =z^{\prime} \zeta Q_{s}^{2}+4 s \cdot\left\{g_{s s}-2 z \zeta Q_{s s}^{2}-4 z \zeta_{s} Q_{s} Q_{s s}-z \zeta_{s s} Q_{s}^{2}\right\} \\
& +2 z \zeta Q_{s} \cdot\left\{4 Q_{s s}+8 s \frac{Q_{s} Q_{s s}}{Q}-4 s \frac{Q_{s}^{3}}{Q^{2}}+4 \frac{Q_{s}^{2}}{Q}+\chi^{(\varepsilon)} Q^{2} Q_{s s}+2 \chi^{(\varepsilon)} Q Q_{s}^{2}\right. \\
& \left.+\chi_{s}^{(\varepsilon)} Q^{2} Q_{s}+2 F_{0} \chi^{(\varepsilon)} Q_{s s}+2 F_{0} \chi_{s}^{(\varepsilon)} Q_{s}\right\} \\
& \leq z^{\prime} \zeta Q_{s}^{2}+4 s \cdot\left\{-2 z \zeta \cdot\left(-\frac{\zeta_{s} Q_{s}}{2 \zeta}\right)^{2}-4 z \zeta_{s} Q_{s} \cdot\left(-\frac{\zeta_{s} Q_{s}}{2 \zeta}\right)-z \zeta_{s s} Q_{s}^{2}\right\} \\
& +2 z \zeta Q_{s} \cdot\left\{4\left(-\frac{\zeta_{s} Q_{s}}{2 \zeta}\right)+8 s \frac{Q_{s}}{Q} \cdot\left(-\frac{\zeta_{s} Q_{s}}{2 \zeta}\right)-4 s \frac{Q_{s}^{3}}{Q^{2}}+4 \frac{Q_{s}^{2}}{Q}\right. \\
& +\chi^{(\varepsilon)} Q^{2} \cdot\left(-\frac{\zeta_{s} Q_{s}}{2 \zeta}\right)+2 \chi^{(\varepsilon)} Q Q_{s}^{2}+\chi_{s}^{(\varepsilon)} Q^{2} Q_{s} \\
& \left.+2 F_{0} \chi^{(\varepsilon)} \cdot\left(-\frac{\zeta_{s} Q_{s}}{2 \zeta}\right)+2 F_{0} \chi_{s}^{(\varepsilon)} Q_{s}\right\} \\
& =z^{\prime} \zeta Q_{s}^{2}-2 s z \frac{\zeta_{s}^{2}}{\zeta} Q_{s}^{2}+8 s z \frac{\zeta_{s}^{2}}{\zeta} Q_{s}^{2}-4 s z \zeta_{s s} Q_{s}^{2}-4 z \zeta_{s} Q_{s}^{2}-8 s z \zeta_{s} \frac{Q_{s}^{3}}{Q} \\
& -8 s z \zeta \frac{Q_{s}^{4}}{Q^{2}}+8 z \zeta \frac{Q_{s}^{3}}{Q}-\chi^{(\varepsilon)} z \zeta_{s} Q^{2} Q_{s}^{2}+4 \chi^{(\varepsilon)} z \zeta Q Q_{s}^{3}+2 \chi_{s}^{(\varepsilon)} z \zeta Q^{2} Q_{s}^{2} \\
& -2 F_{0} \chi^{(\varepsilon)} z \zeta_{s} Q_{s}^{2}+4 F_{0} \chi_{s}^{(\varepsilon)} z \zeta Q_{s}^{2} \text {. }
\end{aligned}
$$


Rearranging this shows that at $\left(s_{0}, t_{0}\right)$ we have

$$
\begin{aligned}
8 s z \zeta \frac{Q_{s}^{4}}{Q^{2}} \leq & \left\{-\frac{8 s z \zeta_{s}}{Q}+\frac{8 z \zeta}{Q}+4 \chi^{(\varepsilon)} z \zeta Q\right\} \cdot Q_{s}^{3} \\
+ & \left\{z^{\prime} \zeta+6 s z \frac{\zeta_{s}^{2}}{\zeta}-4 s z \zeta_{s s}-4 z \zeta_{s}-\chi^{(\varepsilon)} z \zeta_{s} Q^{2}+2 \chi_{s}^{(\varepsilon)} z \zeta Q^{2}\right. \\
& \left.-2 F_{0} \chi^{(\varepsilon)} z \zeta_{s}+4 F_{0} \chi_{s}^{(\varepsilon)} z \zeta\right\} \cdot Q_{s}^{2}
\end{aligned}
$$

or, equivalently,

$$
\begin{aligned}
z \zeta Q_{s}^{2} \leq & \left\{-z \zeta_{s} Q+z \frac{\zeta}{s} Q+\frac{\chi^{(\varepsilon)} z \zeta}{2 s} Q^{3}\right\} \cdot Q_{s} \\
+ & \frac{1}{8} \cdot\left\{z^{\prime} \frac{\zeta}{s} Q^{2}+6 z \frac{\zeta_{s}^{2}}{\zeta} Q^{2}-4 z \zeta_{s s} Q^{2}-4 z \frac{\zeta_{s}}{s} Q^{2}\right. \\
& \left.\quad-\chi^{(\varepsilon)} z \frac{\zeta_{s}}{s} Q^{4}+2 \chi_{s}^{(\varepsilon)} z \frac{\zeta}{s} Q^{4}-2 F_{0} \chi^{(\varepsilon)} z \frac{\zeta_{s}}{s} Q^{2}+4 F_{0} \chi_{s}^{(\varepsilon)} z \frac{\zeta}{s} Q^{2}\right\}
\end{aligned}
$$

Now Lemma 4.1 says that there exists $c_{1}>0$ such that $Q(s, t) \leq c_{1} s^{\frac{2-F_{0}}{4}}$, that is,

$$
Q(s, t) \leq c_{1} s^{\frac{2-\delta}{2}} \quad \text { for all } s \geq 0 \text { and } t \geq 0
$$

in view of (4.10). Notice that $W_{s}^{(\varepsilon)} \geq 0$ implies $Q_{s} \geq 0$. Using (4.12) and the facts that $\chi^{(\varepsilon)} \leq 1$ and $z \leq 1$, on the right of (4.11) we can estimate by Young's inequality

$$
\begin{aligned}
& \left\{z \frac{\zeta}{s} Q+\frac{\chi^{(\varepsilon)} z \zeta}{2 s} Q^{3}\right\} \cdot Q_{s} \leq \frac{1}{4} z \zeta Q_{s}^{2}+z \frac{\zeta}{s^{2}} Q^{2}\left(1+\frac{1}{2} Q^{2}\right)^{2} \\
& \leq \frac{1}{4} z \zeta Q_{s}^{2}+\frac{(4 s)^{\delta}}{s^{2}} \cdot\left(c_{1} s^{\frac{2-\delta}{2}}\right)^{2} \cdot\left(1+\frac{1}{2} \cdot c_{1}^{2} \cdot 2^{2-\delta}\right)^{2} \\
& =\frac{1}{4} z \zeta Q_{s}^{2}+4^{\delta} \cdot c_{1}^{2} \cdot\left(1+\frac{1}{2} \cdot c_{1}^{2} \cdot 2^{2-\delta}\right)^{2},
\end{aligned}
$$

because $\zeta(s) \leq(4 s)^{\delta}$ and we know that $s_{0} \in(0,2)$. Next, we observe that from (4.9) we easily see that $\zeta_{s} \geq 0$ for $s \in\left[0, \frac{3}{2}\right]$, and that since $\delta>1$ there exists $c_{2}>0$ such that

$$
\left|\zeta_{s}(s)\right| \leq c_{2} s^{\delta-1},\left|\zeta_{s s}(s)\right| \leq c_{2} s^{\delta-2} \text { and } \frac{\zeta_{s}^{2}(s)}{\zeta(s)} \leq c_{2} s^{\delta-2} \text { for all } s \in(0,2) \text {. }
$$

Since $Q_{s} \geq 0$, the remaining term $-z \zeta_{s} Q Q_{s}$ obtained from the first bracket in (4.11) is non-positive for $s \leq \frac{3}{2}$, whereas for $s \in\left(\frac{3}{2}, 2\right)$ we again use Young's inequality to find

$$
-z \zeta_{s} Q Q_{s} \leq \frac{1}{4} z \zeta Q_{s}^{2}+z \frac{\zeta_{s}^{2}}{\zeta} Q^{2}
$$


In view of (4.14) and the boundedness of $Q$ we conclude that at all points we have the upper estimate

$$
-z \zeta_{s} Q Q_{s} \leq \frac{1}{4} z \zeta Q_{s}^{2}+c_{3}
$$

for some $c_{3}>0$. In conjunction with (4.12), (4.14) also entails that there exist $c_{4}>0$ and $c_{5}>0$, depending on $\tau$ only, such that

$$
\begin{gathered}
\frac{1}{8} \cdot\left\{z^{\prime} \frac{\zeta}{s} Q^{2}+6 z \frac{\zeta_{s}^{2}}{\zeta} Q^{2}-4 z \zeta_{s s} Q^{2}-4 z \frac{\zeta_{s}}{s} Q^{2}-\chi^{(\varepsilon)} z \frac{\zeta_{s}}{s} Q^{4}-2 F_{0} \chi^{(\varepsilon)} z \frac{\zeta}{s} Q^{2}\right\} \\
\leq c_{4} \cdot\left\{s^{\delta-1} \cdot s^{2-\delta}+s^{2(\delta-1)-\delta} \cdot s^{2-\delta}+s^{\delta-2} \cdot s^{2-\delta}+s^{\delta-2} \cdot s^{2-\delta}\right. \\
\left.+s^{\delta-2} \cdot s^{2(2-\delta)}+0\right\} \leq c_{5}
\end{gathered}
$$

whenever $s \in(0,2)$. Finally, recalling that our requirements on $\chi^{(\varepsilon)}$ were such that $\chi_{s}^{(\varepsilon)} \equiv 0$ on $(\varepsilon, \infty)$ and $\chi_{s}^{(\varepsilon)} \leq \frac{c_{\chi}}{\varepsilon}$, we obtain

$$
\begin{aligned}
& \frac{1}{8} \cdot\left\{2 \chi_{s}^{(\varepsilon)} z \frac{\zeta}{s} Q^{4}+4 F_{0} \chi_{s}^{(\varepsilon)} z \frac{\zeta}{s} Q^{2}\right\} \\
& \leq c_{6} \cdot\left\{\frac{1}{\varepsilon} \cdot \varepsilon^{\delta-1} \cdot \varepsilon^{2(2-\delta)}+\frac{1}{\varepsilon} \cdot \varepsilon^{\delta-1} \cdot \varepsilon^{2-\delta}\right\} \leq c_{7}
\end{aligned}
$$

for all $s \in(0,2)$ with appropriately large $c_{6}>0$ and $c_{7}>0$. All in all, from (4.11), (4.13), (4.15), (4.16) and (4.17) we infer that there exists $c_{8}=c_{8}(\tau)$ such that $g(s, t) \leq c_{7}$ in $(0,2) \times(0, T)$, which since $T>0$ was arbitrary entails that $\zeta Q_{s}^{2} \leq c_{8}$ in $(0,2) \times(\tau, \infty)$. Rewritten in terms of $W^{(\varepsilon)}$, this means that

$$
\begin{aligned}
\left(W_{s}^{(\varepsilon)}(s, t)\right)^{2} & =4 Q^{2}(s, t) Q_{s}^{2}(s, t) \leq 4 c_{8} \cdot \frac{1}{\zeta(s)} \cdot Q^{2}(s, t) \\
& \leq 4 c_{8} \cdot s^{-\delta} \cdot c_{1}^{2} s^{2-\delta}=4 c_{1}^{2} c_{8} s^{2-2 \delta} \quad \text { for all } s \in(0,1] \text { and } t>\tau,
\end{aligned}
$$

because $\zeta(s) \geq s^{\delta}$ for all $s \in(0,1]$. As $2-2 \delta=-F_{0}$ by (4.10), this shows the validity of (4.7) for $s \in(0,1]$ and $t>\tau$ and thereby completes the proof.

Proof of Theorem 0.2ii). The statement is an immediate consequence of Lemma 4.2.

\section{References}

[1] P. BILER, Local and global solvability of some parabolic systems modelling chemotaxis, Adv. Math. Sci. Appl. 8 (1998), 715-743.

[2] P. BILER, Radially symmetric solutions of a chemotaxis model in the plane - the supercritical case, In: "Parabolic and Navier-Stokes Equations", Banach Center Publications, Polish Acad. Sci., Warsaw, Vol. 81, 2008, 31-42. 
[3] P. BILER and G. KARCH, Blowup of solutions to generalized Keller-Segel model, J. Evol. Equ. 10 (2010), 247-262.

[4] P. Biler, G. KARCh, P. LAurençOT and T. NADZieja, The $8 \pi$-problem for radially symmetric solutions of a chemotaxis model in a disc, Topol. Methods Nonlinear Anal. 27 (2006), 133-147.

[5] P. BILER and T. NADZIEJA, An elementary approach to nonexistence of solutions of linear parabolic equations, Colloq. Math. 122 (2011), 125-134.

[6] A. Blanchet, J. Dolbeault and B. Perthame, Two-dimensional Keller-Segel model: Optimal critical mass and qualitative properties of the solutions, Electron. J. Differential Equations 2006 (2006), no. 44, 133.

[7] A. Blanchet, J. A. Carrillo and N. Masmoudi, Infinite Time Aggregation for the Critical Patlak-Keller-Segel model in $\mathbb{R}^{2}$, Comm. Pure Appl. Math. 61 (2008), 1449-1481.

[8] S. ChILdress and J. K. Percus, Nonlinear aspects of chemotaxis, Math. Biosci. 56 (1981), 217-237.

[9] H. GAJEWSKI and K. ZACHARIAS, Global behaviour of a reaction-diffusion system modelling chemotaxis, Math. Nachr. 195 (1998), 77-114.

[10] V. A. GalaKtionov and J. L. VÁZQUeZ, Continuation of blow-up solutions of nonlinear heat equations in several space dimensions, Comm. Pure Appl. Math. 50 (1997), 1-67.

[11] M. A. Herrero and J. J. L. VelázQUeZ, Singularity patterns in a chemotaxis model, Math. Ann. 306 (1996), 583-623.

[12] M. A. Herrero and J. J. L. VelázQuez, A blow-up mechanism for a chemotaxis model, Ann. Sc. Norm. Super. Pisa, Cl. Sci. (4) 24 (1997), 633-683.

[13] T. Hillen and K. J. PAINTER, A user's guide to PDE models for chemotaxis, J. Math. Biol. 58 (2009), 183-217.

[14] D. Horstmann, From 1970 until present: The Keller-Segel model in chemotaxis and its consequences. I, Jahresber. Deutsch. Math.-Verein. 105 (2003), 103-165.

[15] D. HORSTMANN and G. WANG, Blow-up in a chemotaxis model without symmetry assumptions, European J. Appl. Math. 12 (2001), 159-177.

[16] W. JÄGER and S. LUCKHAUS, On explosions of solutions to a system of partial differential equations modelling chemotaxis, Trans. Amer. Math. Soc. 329 (1992), 819-824.

[17] E. F. KELLER and L. A. SEGEL, Initiation of slime mold aggregation viewed as an instability, J. Theor. Biol. 26 (1970), 399-415.

[18] E. F. KelLER and L. A. SEgEL, Model for chemotaxis, J. Theoret. Biol. 30 (1971), 225234.

[19] R. KowalczyK, Preventing blow-up in a chemotaxis model, J. Math. Anal. Appl. 305 (2005), 566-588.

[20] M. KUROKIBA and T. OGaWA, Finite time blow-up of the solution for a nonlinear parabolic equation of drift-diffusion type, Differ. Integral Equations 16 (2003), 427-452.

[21] D. A. LadYẑEnskaja, V. A. Solonnikov and N. N. URAltseVa, "Linear and Quasilinear Equations of Parabolic Type", Translations of Mathematical Monographs, Vol. 23, Amer. Math. Soc., Providence, RI, 1968.

[22] T. NAGAI, Blowup of radially symmetric solutions to a chemotaxis system, Adv. Math. Sci. Appl. 5 (1995), 581-601.

[23] T. NAGAI, Blow-up of non-radial solutions to parabolic-elliptic systems modeling chemotaxis in two-dimensional domains, J. Inequal. Appl. 6 (2001), 37-55.

[24] T. NagAi, T. SenBA and K. Yoshida, Application of the Trudinger-Moser inequality to a parabolic system of chemotaxis, Funkcial. Ekvac. 40 (1997), 411-433.

[25] Y. NAITO and T. SUZUKI, Self-Similar solutions to a nonlinear parabolic-elliptic system, Taiwanese J. Math. 8 (2004), 43-55.

[26] C. S. PATLAK, Random walk with persistence and external bias, Bull. Math. Biophys, 15 (1953), 311-338.

[27] P. Quittner and PH. Souplet, "Superlinear Parabolic Problems: Blow-up, Global Existence and Steady States", Birkhäuser, Basel, Boston, Berlin, 2007. 
[28] T. Senba and T. Suzuki, Parabolic system of chemotaxis: blowup in a finite and the infinite time, Methods Appl. Anal. 8 (2001), 349-367.

[29] T. SENBA and T. SUZUKI, Chemotactic collapse in a parabolic-elliptic system of mathematical biology, Adv. Differential Equations 6 (2001), 21-50.

[30] T. SENBA and T. SUZUKI, Weak solutions to a parabolic-elliptic system of chemotaxis, J. Funct. Anal. 191 (2002), 17-51.

[31] T. SENBA and T. SuzUKI, Chemotactic collapse of radial solutions to Jäger-Luckhaus system, Adv. Math. Sci. Appl. 14 (2004), 241-250.

[32] T. SuZUKI, "Free Energy and Self-Interacting Particles", Birkhäuser, Boston, 2005.

[33] T. SuZuKi and T. SEnBA, "Applied Analysis - Mathematical Methods in Natural Science", Second Edition, Imperial College Press, London, 2011.

[34] J. J. L. VELÁZQUEZ, Stability of some mechanisms of chemotactic aggregation, SIAM J. Appl. Math. 62 (2002), 1581-1633.

[35] J. J. L. VelázQueZ, Point dynamics in a singular limit of the Keller-Segel model.I. Motion of the concentration regions, SIAM J. Appl. Math. 64 (2004), 1198-1223.

[36] J. J. L. VelázQuez, Point dynamics in a singular limit of the Keller-Segel model. II. Formation of the concentration regions, SIAM J. Appl. Math. 64 (2004), 1224-1248.

[37] W. WAGNER et al., Hematopoietic progenitor cells and cellular microenvironment: behavioral and molecular changes upon interaction, Stem Cells 23 (2005), 1180-1191.

Departamento de Matemática Aplicada

EU Informática

Universidad Politécnica de Madrid

28031 Madrid, Spain

jtello@eui.upm.es

Institut für Mathematik

Universität Paderborn

33098 Paderborn, Germany

michael.winkler@math.uni-paderbon.de 